\title{
EFFECT OF REMEDIAL AND AMENDMENTS USES ON SOME BOTANICAL CHARACTRISTICS AND YIELD OF PEA AND sunflower PLANTS Pisum sativum $L$. and Helianthus annus $L$. El-Emary , F. A.A ${ }^{-1}$.and N.I.A. Talha ${ }^{-2}$ \\ 1. Dept. of Agric. Botany, Fac. of Agric., Assiut, Al-Azhar Univ. Egypt. \\ 2. Soils, Water and Environ. Res. Inst.Sakha Agric., Res. Station, Egypt.
}

\begin{abstract}
Two field experiments were carried out at Sakha Experimental Station, Kafr ElSheikh Governorate during the two growing seasons, of 2012 and 2013 The effects of sewage sludge (SS), poultry manure (PM), processed town refuse (PTR), mixture of SS and PM, recommended rates of NPK, mineral fertilizers (RMF) without or with gypsum, or sulphur, on the certain Botanical characteristics and yield of pea plants were studied. In addition, the residual effects on the yield and its components of sunflower plants were investigated. Remedial and amendments were incorporated into the plowing soil $(0-15 \mathrm{~cm})$ before planting pea plants.

The obtained data can be summarized as follows:

1-D.W and Seed index were markedly increased by increasing the biosolids application rate. The application of $\mathrm{SS}_{5}$ (without) agrochemicals, $\mathrm{PM}_{2}$ with gypsum and $\mathrm{PM}_{2}$ with sulphur produced the highest Dry weight.

2-Concentration of plastid pigments in the pea leaves were increased due to the application of $\left(\mathrm{SS}_{5}\right)$ and sulphur. Addition of remedial with or without agrochemicals increased, N, P, K, Cu, Mn, Zn. Heavy metals, $\mathrm{Cd}, \mathrm{Pb}$ and $\mathrm{Ni}$ contents of straw were also increased compared with the control but less than the toxic limits for these plants.

3-SS ${ }_{5}$, without; $(\mathrm{SS}+\mathrm{PM})_{3}$ and with gypsum and PM2 with sulphur increased seed yield if compared to control. The application of $\mathrm{SS}_{5}$ without agrochemicals, $\mathrm{PM}_{2}$ with gypsum and $\mathrm{PM}_{2}$ with sulphur produced the highest straw yield.

The residual effects due to remedial and amendments treatments showed that:-

4-Plant height; D.W were increased due to pre-incorporated (SS+PM) $)_{3}$. Similarly, stem and head diameter recorded, highest values by $\mathrm{PM}_{2}$ gypsum and sulphur in a descending order.

5-Plastid pigments Macro, Micro and Heavy metals, were also increased. The highest values plastid pigments were obtained in sulphur-sewage sludge pre-treated soils at the high levels. At the same time N, P and $\mathrm{K}$ content of different sunflower plants organs were higher in $\mathrm{SS}_{5},(\mathrm{SS}+\mathrm{PM}) 3$ and PM2 respectively, in soil than those obtained in low rate of organic manure treatments.

6-The highest seed oil yield was obtained due to $\left(\mathrm{PM}_{2}\right.$ and $\left.\mathrm{SS}_{3}\right)$ without application.

It could be concluded that remedial and amendments, i.e, $\mathrm{SS}_{5}$, (without); PM2(with gypsum) and PM2 (with sulphur) have a significant influence on Botanical characteristics and yield of pea plants. Their residual effects extends to best botanical characteristics and yield of sunflower plants under the experimental condition.

Keywords: Botanical characteristic, remedial, amendments, pea, sunflower.
\end{abstract}

\section{INTRODUCTION}

How do you get best Botanical characteristics, highest yield and quality by using available materials under our Egyptian condition? There is no doubt the mineral fertilizers are essential in most cropping systems, if maximum yields are to be realized. It caused adverse effects on public health and the environment from the absence of waste management programmes in rural and urban areas. 
Now days, the growers tended to use the natural sources of fertilizers and conditioners via using the sewage sludge and poultry manures as well as amendments; gypsum and sulphur, (Lorenz and_Maynard, 1980; Mengel and Kirkby, 1987; Hilal et al, 1990 and Raven and Loeppert, 1997)

Land application of sulphur to soil has an important role not only for chemical amelioration of alkaline soil, but also as fertilizer. Moreover, it plays several important roles in soils such as reducing soil $\mathrm{pH}$, providing $\mathrm{SO}_{4}{ }^{=}$to plants, and increasing availability of some nutrients._Recently, municipal sewage sludge has become more attractive for agriculture utilization because this material is characteristically high in the major plant nutrients $\mathrm{N}$ and $\mathrm{P}$. However, this material may also contain high concentrations of heavy metals including $\mathrm{Cd}, \mathrm{Zn}, \mathrm{Cu}$ and other metals. There are few potentially toxic metals most likely to occur in sewage sludge and therefore, would be of our interest in protecting the food chain in agriculture systems; in particular $\mathrm{Cd}, \mathrm{Cu}, \mathrm{Ni}, \mathrm{Pb}$ and $\mathrm{Zn}$ to a lesser extent As, Co, Mn and Mo (Alina et al., 1995).

Legumes are also capable of symbiotic nitrogen fixation enriching the soil condition suitable for both mix and alternate cropping (Bromfiled,2001), a true multifunctional crop that may be used as green forage, forage dry matter, forage meal, silage, haylage, immature grain, straw and green manure (Mikic et al., (2011). Arisha and Abd El-Bary (2000) found that application of sulphur had no significant effect, neither on growth or yield of pea and spinach; while sewage sludge application significantly increased growth and yield of both crops. Mashaly et al. (1993) found that the contents of NPK were increased in bean shoots and grains with increasing rate of sewage sludge addition. However the concentration of $\mathrm{Mn}, \mathrm{Zn}, \mathrm{Cu}, \mathrm{Ni}, \mathrm{Pb}, \mathrm{Co}, \mathrm{Hg}$ and $\mathrm{Cr}$ were not significantly increased

Sunflower (Helianthus annus. L.) belonging to the family Asteraceae, is a major oil seed, used four important annual crops in the world for edible oil. Seeds of Sunflower contain 24-49\% oil (Saleh et al.,2008). Omar and Abou Bakr (1991) studied the effect of three organic residues, e.g. fresh garbage, fresh compost and matured compost with or without NPK fertilizers on growth, yield and yield components, seed oil content and oil quality of sunflower cv. Mayak, The tested parameters were significantly increased due to application of different mineral fertilizers and/or organic materials added. .Application of NPK fertilizers through all sources was found by many authors to enhance yield quantitively and qualitatively of field crops Allievei et al., 1993; Preer et al., 1995; Baca et al., 1995; Mahmoud et al., 2001; Saleh et al.,2004; and Mohamed 2012.

\section{The present study aimed to study the following:-}

(a)The potential effects of different levels and types of remedial uses, e.g., sewage sludges (SS); poultry manures (PM) and processed town refuse (PTR) single or in combined additions (SS + PM) with or without some amendments conditioners, e.g., sulphur and gypsum on the botanical characteristics of pea plants (Morphological , physiological and yield)

(b)The residual effects of the treatment used on Botanical characteristics and yield of sunflower plants. 


\section{MATERIALS AND METHODS}

Field experiments :

Two successive field experiments were assessed at the Experimental Farm of Sakha Agricultural Research Center. After harvesting the proceeding wheat crop, representative composite surface soil samples $(0-30 \mathrm{~cm})$ were collected, air-dried and passed through a $2 \mathrm{~mm}$ sieve. Thoroughly mixed samples were analyzed for their soil characteristics and $\mathrm{N}, \mathrm{P}, \mathrm{K}$ contents (Table 1) according to Page (1982). Available $\mathrm{Cu}, \mathrm{Mn}, \mathrm{Zn}, \mathrm{Cd}, \mathrm{Pb}$ and Ni were extracted by DTPA according to Lindsay and Norvell, (1978) in soil and organic manures. Also, total content of above elements in soil and organic manures were digested using aqua regia Cottenie et al., (1982). Both total and available of tested lements were measured using atomic absorption spectrophotometericalley (Perkin Elimer, 3300).The texture of experimental soil was clay (sand 21.5, silt 31.6 and clay $46.9 \%$ ) with water table depth of $110 \mathrm{~cm}$.

The two experiments were carried out in the same area as the first crop was pea (Winter, 2012) and after harvesting, the same experimental plots were cultivated with sunflower (summer, 2013) in a split plot design with four replicate. The main three plots were devoted to the agrochemicals treatments, i.e., none, gypsum $\left(5 \mathrm{Mg} \mathrm{fed}^{-1}\right)$ and sulphur $\left(400 \mathrm{~kg} \mathrm{fed}^{-1}\right)$ and the sub-plots were eleven, which can be summarized as follows:

\begin{tabular}{|c|c|c|c|c|c|}
\hline & & & Ince & orporated materials & \\
\hline No. & Treatment & & No. & Treatment & \\
\hline 1 & Control * & C & 6 & Processed town refuse, $1 \%$ & $\mathrm{PTR}_{1}$ \\
\hline 2 & $\begin{array}{c}\text { Sewage sludge, } \\
3 \%\end{array}$ & $\mathrm{SS}_{3}$ & 7 & Processed (own refuse, $2 \%$ & $\mathrm{PTR}_{2}$ \\
\hline 3 & $\begin{array}{c}\text { Sewage sludge, } \\
5 \%\end{array}$ & $\mathrm{SS}_{5}$ & 8 & $\begin{array}{c}\text { (Sewage sludge + poultry manure, } \\
1: 1), 1 \%\end{array}$ & $(\mathrm{SS}+\mathrm{PM})_{1}$ \\
\hline 4 & $\begin{array}{c}\text { Poultry manure, } \\
1 \% \\
\end{array}$ & PMi & 9 & $\begin{array}{c}\text { (Sewage sludge + poultry manure, } \\
1: 1), 2 \%\end{array}$ & $(\mathrm{SS}+\mathrm{PM})_{2}$ \\
\hline 5 & $\begin{array}{c}\text { Poultry manure, } \\
2 \%\end{array}$ & $\mathrm{PM}_{2}$ & 10 & $\begin{array}{c}\text { (Sewage sludge + poultry manure, } \\
1: 1), 3 \%\end{array}$ & $(\mathrm{SS}+\mathrm{PM})_{3}$ \\
\hline & & & 11 & $\begin{array}{l}\text { recommended rates of mineral } \\
\text { fertilizers ** }\end{array}$ & RMF \\
\hline
\end{tabular}

\section{Sources and forms of remedial to (organic manures) and (agrochemical) amendment USES:}

Air dried composite subsamples of anaerobically digested SS, i.e., remedial uses, were collected from accumulated deposits at the municipal sewage treatment plant at Kafr El-Sheikh after the secondary-treatments. Subsamples of PM were obtained from the experimental college (Agric. Kafr El-Sheikh) farm. Both SS and PM dried materials were spreader on clean plastic sheets under sunlight for one week while being covered with plastic sheets to enhance photosensitization. Subsamples of PTR amendment were obtained from Al-Mansoura composting Factory. The obtained organic amendments were air dried, pulverized and passed through a $2 \mathrm{~mm}$ sieve 
and then analyzed for their initial chemical characteristics (Table 1). Urea $(46.5 \% \mathrm{~N})$ and superphosphate $\left(15.5 \% \mathrm{P}_{2} \mathrm{O}_{5}\right)$ were used as nitrogen and phosphorus fertilizers. All the experimental plots received $20 \%$ of $\mathrm{P}$ (during cultivation) and $\mathrm{N}$ (at sowing) as starter regard to RMF. The rest $80 \%$ of the recommended $\mathrm{P}$ were also added during cultivation for the RMF treatments, i.e., conventional farming.

Table (1):Some characteristics of the tested soil, remedial and amendment uses.

\begin{tabular}{|c|c|c|c|c|c|c|c|c|}
\hline Tested characteristics & \multicolumn{2}{|c|}{ Soil } & \multicolumn{2}{|c|}{ SS } & \multicolumn{2}{|c|}{ PM } & \multicolumn{2}{|c|}{ PTR } \\
\hline $\mathrm{pH}(1: 2.5)$ soil : water. & \multicolumn{2}{|c|}{7.98} & \multicolumn{2}{|c|}{6.25} & \multicolumn{2}{|c|}{7.11} & \multicolumn{2}{|c|}{7.69} \\
\hline $\mathrm{EC}_{\mathrm{e}}^{*}$ at $25^{\circ} \mathrm{C}, \mathrm{dSm}^{-1}$ & \multicolumn{2}{|c|}{3.30} & \multicolumn{2}{|c|}{$3.15^{\star *}$} & \multicolumn{2}{|c|}{$4.31^{\star *}$} & \multicolumn{2}{|c|}{$7.30^{\star *}$} \\
\hline SP (saturation percent), \% & \multicolumn{2}{|c|}{78.10} & \multicolumn{2}{|c|}{182.88} & \multicolumn{2}{|c|}{158.76} & \multicolumn{2}{|c|}{121.86} \\
\hline TN (total nitrogen), \% & \multicolumn{2}{|c|}{0.07} & \multicolumn{2}{|c|}{2.06} & \multicolumn{2}{|c|}{3.05} & \multicolumn{2}{|c|}{0,99} \\
\hline TOC, ${ }^{\star * \star} \%$ & \multicolumn{2}{|c|}{0.72} & \multicolumn{2}{|c|}{28.26} & \multicolumn{2}{|c|}{32.33} & \multicolumn{2}{|c|}{27.79} \\
\hline OM (organic matter) \% & \multicolumn{2}{|c|}{1.24} & \multicolumn{2}{|c|}{48.60} & \multicolumn{2}{|c|}{55.60} & \multicolumn{2}{|c|}{47.80} \\
\hline $\mathrm{C} / \mathrm{N}$ ratio & \multicolumn{2}{|c|}{10.23} & \multicolumn{2}{|c|}{13.73} & 10. & .59 & & 7.96 \\
\hline Soluble cations ${ }^{*} \mathrm{meq} / \mathrm{L}$ & & & & & & & & \\
\hline $\mathrm{Ca}^{++}$ & & .05 & & 50 & 6.3 & & & 9.68 \\
\hline $\mathrm{Mg}^{++}$ & & 69 & & 24. & 20. & 16 & & 1.72 \\
\hline $\mathrm{Na}^{+}$ & & .90 & & 20 & 6.5 & 59 & & .97 \\
\hline $\mathrm{K}^{+}$ & & 83 & & 58 & 10. & 10 & & .58 \\
\hline Soluble anions* $\mathrm{meq} / \mathrm{L}$ & & & & & & & & \\
\hline $\mathrm{CO}_{3}{ }^{=}$ & & 00 & & .00 & 0.0 & 00 & & .00 \\
\hline $\mathrm{HCO}_{3}^{-}$ & & 90 & & 50 & 5.1 & 10 & & 1.50 \\
\hline $\mathrm{Cl}^{-}$ & & .10 & & 10 & 30. & 12 & & .30 \\
\hline $\mathrm{SO}_{4}=$ & & 15 & & .22 & 7.8 & 89 & & .16 \\
\hline Available, $\mathrm{mg} \mathrm{kg}^{-1}$ & & & & & & & & \\
\hline $\mathrm{N}$ & & .50 & & 1.20 & 1071 & 1.00 & & 0.00 \\
\hline$P$ & & 77 & & 65 & 120 & .32 & & 1.52 \\
\hline $\mathrm{K}$ & 392 & 2.50 & 220 & 0.00 & 537 & .20 & & \\
\hline DTPA and total, $\mathrm{mg} \mathrm{kg}^{-1}$ & DTPA & Total & DTPA & Total & DTPA & Total & DTPA & Total \\
\hline $\mathrm{Zn}$ & 0.38 & 72.0 & 37.14 & 343.20 & 6.40 & 54.20 & 32.50 & 291.00 \\
\hline $\mathrm{Mn}$ & 7.80 & 427.2 & 43.50 & 395.80 & 3.69 & 35.60 & 34.04 & 290.80 \\
\hline $\mathrm{Cu}$ & 1.42 & 52.6 & 26.24 & 175.80 & 4.9 & 38.00 & 9.94 & 127.00 \\
\hline $\mathrm{Pb}$ & 0.52 & 70.2 & 18.22 & 251.80 & 8.89 & $8 \mathrm{i}: 20$ & 10.84 & 190.20 \\
\hline $\mathrm{Cd}$ & 0.02 & 1.2 & 0.36 & \begin{tabular}{|l|}
4.40 \\
\end{tabular} & 0.31 & 3.40 & 0.45 & 3.00 \\
\hline $\mathrm{Ni}$ & 0.02 & 3.7 & 1.12 & 70.20 & 0.27 & 22.80 & 0.66 & 49.60 \\
\hline
\end{tabular}

\section{Agriculture practices:}

Seeds of pea $35 \mathrm{~kg} \mathrm{fed}^{-1}$,(Pisum sativum L.), cv. Progress No 9, (from Agriculture Research Center) were drilled into soil on December, $5^{\text {lh }}, 2012$ and harvested in May, $5^{\text {th }}, 2013$. The experimental plot area was $15 \mathrm{~m}^{2}(3 \mathrm{x}$ 5) $\mathrm{m}$ and consisted of 5 rows with $60 \mathrm{~cm}$ spacing. Added biosolid and agrochemicals were incorporated into the soil $(0-15 \mathrm{~cm})$ before planting. Seeds inoculation' with infective and effective of strain Rhizobium leguminosarum biovar. The rest $80 \%$ of mineral $\mathrm{N}$, i.e., $56 \mathrm{~kg} \mathrm{fed}^{-1}$ urea- $\mathrm{N}$ 
was added in four equal doses. The first dose was added 18 days after sowing before the first irrigation, while the other three splits, where added each 15 days. After 75 days of plant growths, plants collected; from each plot were hand pulled at random to assess the mean heights, as well as botanical chracrisitis and yield components of each plots.

Five $\mathrm{kg} \mathrm{fed}^{-1}$ of sunflower seeds (Helianthus annus L.), cv. Giza 1 were grown on the same soil plots of the harvested pea crop by sowing in the last week of May during 2013 on hills, 3-4 seeds/hill, with $30 \mathrm{~cm}$ spacing and 50 $\mathrm{cm}$ wide ridges. After 21 days of germination (2-4 leaf stage), plants were thinned to one plants per hill and $48 \mathrm{~kg} \mathrm{fed}^{-1}$ of urea- $\mathrm{N}$ was added. The first fraction, $24 \mathrm{~kg} \mathrm{~N}$.was dressed after thing and the second, $24 \mathrm{~kg} \mathrm{~N}$. was two weeks later. sunflower plants were harvested in the last week of Sep.2013 and the mean of following assessments of botanical characteristics and yield components were determined.

Botanical characteristics and yield components :-

At harvest a sample of five plants from every plot were chosen at random to measure the following characters:-

A-Morphlogical characteristics of pea and sunflower i.e, dry weight (D.W) $(\mathrm{g}) /$ plant,Seed index weight $100(\mathrm{~g})$ and Plant height $(\mathrm{cm})$, Stem diameter $(\mathrm{cm})$, Head diameter $(\mathrm{cm})$, D.W stem $(\mathrm{g}) /$ plant and D.W leaves $(\mathrm{g}) /$ plant were recorded

B-Physiologicai characteristics of pea and sunflower, plastid pigments(chl.a, chl.b, total chl and carotenoids $\mathrm{mg} / \mathrm{g}$ F.w leaves) according to Inskeep, and Bloom (1985): as well as Macro, Micro and heavy metal (N, P, K, Cu, Mn, Zn, $\mathrm{Cd}, \mathrm{Pb}$ and $\mathrm{Ni}$ content in plant organs according to Snell and Snell (1977) were estimated.

c- Yield and its components of pea and sunflower including Seed yield (g) / plant, Seed yield $(\mathrm{mg})$ fed $^{-1}$ and Straw yield $(\mathrm{kg}) \mathrm{fed}^{-1}$, Seed yield $(\mathrm{g}) / \mathrm{plant}, 100$ Seed weight $(\mathrm{g})$, Seed yield $(\mathrm{kg}) \mathrm{fed}^{-1}$, Oil yield $(\mathrm{kg})$ fed $^{-1}$ and Oil \% in seeds were evaluated.

Collected pea and sunflower plants were washed with tap and distilled water, oven dried at $70^{\circ} \mathrm{C}$ for 24 hours, weighed, and ground in a stainless steel mill. To assess protein in both straw and seed materials, $(0.5 \mathrm{~g})$ were digested according to Chapman and Pratt, (1961). The obtained digestion solution were used to find out: The total-concentrations of $\mathrm{N}, \mathrm{P}$ and $\mathrm{K}\left(\mathrm{g} \mathrm{kg}^{-1}\right)$ after Page (1982). $\mathrm{Cu}, \mathrm{Mn}, \mathrm{Zn}, \mathrm{Cd}, \mathrm{Pb}$ and $\mathrm{Ni}\left(\mathrm{mg} \mathrm{kg}^{-1}\right)$ were determined by atomic absorption spectrophotometers as mentioned above. The oil yield and percentage in sunflower seeds were determined according to AOAC (1980). The statistical analysis was done by using computer program. Data were tested by analysis of variance Duncans Multiple range test used to comparisons a many treatments means (Duncan, 1955). All statistical analysis were performed using analysis of variance technique by means of IRRISTAT Computer soft ware package. 


\title{
RESULTS AND DISCUSSION
}

\author{
Effect of remedial and amendments uses on some botanical \\ characteristics to pea plants \\ Morphological characteristics: \\ Data presented in Table (2) showed highly significant differences in
} morphological characteristics to pea plants which were higher in soils received organic manures used as single or co-organic waste substrates than these obtained from control. Assessed parameters also showed the same trend with applied RMF but the increase was significantly less than with organically treated soils. The addition of biosolids and/or agrochemicals significantly increased $D$. W (g) / plant and Seed index Weight 100(g).. D. W (g) / plant and Seed index Weight $100(\mathrm{~g})$ were markedly increased by increasing the biosolids application rate.

Data in Table (2) showed that the morphological with $\mathrm{SS}_{5}$, without added agrochemicals, $(\mathrm{SS}+\mathrm{PM})_{3}$ with gypsum and $\mathrm{PM} 2$ with sulphur was increased compared with the control. The application of $\mathrm{SS}_{5}$ without agrochemicals, $\mathrm{PM}_{2}$ with gypsum and $\mathrm{PM}_{2}$ with sulphur produced the highest straw yield. The values were 15, 18 and 23.75 D. W (g) / plant, respectively.

The obtained results are in agreement with the findings of_El-Saady (1991); Fatma Osman (1998) and Negm et al. (1998). They reported that application of sewage sludge at different rates, progressively and significantly increased the straw dry; seeds yield and fresh and dry yield of pea crop. Gendy et al. (1996) showed that soybean nodules, number and their dry weight responsed significantly to both gypsum and phosphate application alone or together. as well as positive effects on green pod yield and harvest index.

\section{Physiological charactristics: plasted pigments content:}

Data in Table (3) show that, values of. total chl. ranged between 4.40 to $7.35,4.49$ to 7.64 and 4.96 to 7.63 (mg $/ \mathrm{g} \mathrm{F.w}$ for none, gypsum and sulphur treatments, respectively. In general, incorporated $\mathrm{SS}_{5}$ and $\mathrm{SS}_{3}$ without any agrochemicals, with gypsum or sulphur gave the Plastid pigments leave plants (Table 3). The above results are in harmony with those of ElHindi et al. (2000) and Mohamed and Kandeel (1998). Table (3) also shows that highly significant interaction effect between agrochemicals (AC) and organic manures (B), i.e., (AC $\times \mathrm{B}$ ) on Plastid pigments in all treatments. With regard to the effect of biosolids, data emphasize that mixing $\mathrm{SS}_{5}$ and $\mathrm{SS}_{3}$ led to a significant increase with regard to the tested parameters over the control treatments. This might be due to the synergetic effect between SS5 and PM2 which has more contents of both total and active $\mathrm{N}$ and $\mathrm{P}$ (Table 4).

\section{Macronutrients content:}

Data listed in Table (4) revealed that, the N, P and K contents of pea plants organs were markedly increased due to the application of the different types and rates of organic manures and RMF as compared to the control. The contents of $\mathrm{N}, \mathrm{P}$ and $\mathrm{K}$ of PEA seed were higher than straw. 
J. Plant Production, Mansoura Univ., Vol. 5(12), December, 2014 2

2111 
EI-Emary, F. A.A $A^{-1}$ and N.I.A. Talha ${ }^{-2}$

3

2112 
J. Plant Production, Mansoura Univ., Vol. 5(12), December, 2014

4

2113 
Also, data in Table (4) show that the $\mathrm{N}$ and $\mathrm{P}$ contents of PEA seed and straw were higher under application of RMF than those obtained with low rates of organic manures, i.e., $\left(\mathrm{PM}_{1} ; \mathrm{PTR}_{1}\right)$ and $(\mathrm{SS}+\mathrm{PM})_{1}$. However, applied or co-applied higher rates of biosolids, i.e., 47.05; 47.50and47.78 and 4.85;5.15 and5.05 and11.00; 11.58 and 11.38 increased N, P and K contents of seed, respectively. the proposed treatments led to higher contents of assessed elements with marked effect of added sulphur than gypsum. Coapplication of organic manures at any level, increased the $\mathrm{N}, \mathrm{P}$ and $\mathrm{K}$ contents of PEA seed and straw than those applied individually at the same rates which may be due to their synergetic or co-metabolizing effects.

Clearly the beneficial effects of sulphur by Mohamed and Kandeel (1998) of pea and sulphur and sewage sludge application on growth, productivity, the content of macro-nutrients, and heavy metals in the edible parts of spinach and pea as well as in the soil at the end of the experiments. The results indicated that application of sulphur had no significant effect, neither on growth or yield in both crops Arisha and Abd El-Bary (2000) add to Mashaly et al., (1993) in bean shoots and grains.

\section{Micronutrients content:}

Results recorded in Tables (5 and 6) show that, $\mathrm{Cu}, \mathrm{Mn}, \mathrm{Zn}, \mathrm{Cd}, \mathrm{Pb}$ and $\mathrm{Ni}$ contents of different PEA organs were higher for application of organic manures to soil than those obtained with RMF.

Addition of gypsum to soil led to higher contents of micronutrients ( $\mathrm{Zn}$ and $\mathrm{Mn}$ ) of different PEA organs than those obtained with sulphur treated soil, i.e. gypsum > sulphur > none. Also, data show that $\mathrm{Cu}, \mathrm{Cd}, \mathrm{Ni}$ and $\mathrm{Pb}$ elements of different pea organs (seed and straw) with sulphur treated soil were higher than those obtained with gypsum treated soil compared with the none agrochemicals treated soils.

Results in Tables (5 and 6) indicate also that, values of $\mathrm{Zn}, \mathrm{Mn}$ and $\mathrm{Cu}$ of different organs showed the following decreasing orders in seed and straw: $\mathrm{Zn}>$ $\mathrm{Mn}>\mathrm{Cu}$. The magnitude values of $\mathrm{Cd}, \mathrm{Ni}$ and $\mathrm{Pb}$ of different flax organs exhibited the following decreasing order: straw > seed. Mixtures (1: 1) of organic manures added at any level, led to a decrease in the microelements and heavy metals content of different organs than those obtained by the same rates of the added individual organic manures, which may be due to the dilution effect of added materials.

The abovementioned data Tables (5 and 6) show clearly the beneficial effects of gypsum and sulphur on quality of the different PEA organs as they increased nutrients content with regard to control. Generally under the conditions of investigation although the combined organic manures with gypsum and sulphur were more pronounced in increasing the concentrations of studied heavy metals, which were still low and less than the recorded detrimental limits found in plants as given by Kabate-Pendias and Pendias (1992) and Grazabaky et al., (1992). These results indicate the importance of studying heavy metals (Cd, Ni and $\mathrm{Pb}$ ) specially, bioaccumulation and cycling in the environment for management of agricultural soils and crops. 
J. Plant Production, Mansoura Univ., Vol. 5(12), December, 2014

5

2115 
El-Emary, F. A.A $A^{-1}$ and N.I.A. Talha ${ }^{-2}$

6

2116 


\section{Heavy metals content:}

The above mentioned data in Tables (5 and 6 ) show clearly the beneficial effects of gypsum and sulphur on quality of the different PEA organs as they increased nutrients content with regard to control. Generally under the conditions of investigation although the combined organic manures with gypsum and sulphur were more pronounced in increasing the concentrations of studied heavy metals, which were still low and less than the recorded detrimental limits found in plants as given by Kabate-Pendias and Pendias (1992) and Grazabaky et al., (1992). These results indicate the importance of studying heavy metals $(\mathrm{Cd}, \mathrm{Ni}$ and $\mathrm{Pb}$ ) specially, bioaccumulation and cycling in the environment for management of agricultural soils.

\section{Yield characteristics:}

The addition of biosolids and/or agrochemicals significantly increased seed and straw yield. Seed and straw yield are also affected by types and rates of biosolids. Seed yield of pea/Mg kg-1 was markedly increased by increasing the biosolids application rate.

Table (7):Yield characteristics of pea plants as affected by remedial and amendments

\begin{tabular}{|c|c|c|c|c|c|c|c|c|c|}
\hline \multirow{3}{*}{ Treat. } & \multicolumn{9}{|c|}{ Agrochemicals uses } \\
\hline & None & $\mathbf{G}$ & $\mathrm{S}$ & None & $\mathbf{G}$ & $\mathbf{S}$ & None & $\mathbf{G}$ & $\mathbf{S}$ \\
\hline & \multicolumn{3}{|c|}{ Seed yield (g) / plant } & \multicolumn{3}{|c|}{ Seed yield $(\mathrm{mg})$ fed $^{-1}$} & \multicolumn{3}{|c|}{ Straw yield $(\mathrm{kg}) \mathrm{fed}^{-1}$} \\
\hline Control & $20.60 \mathrm{e}$ & 24.14 ef & $25.63 e$ & $2.42 f$ & $2.64 f$ & $2.71 \mathrm{~g}$ & $1.56 \mathrm{e}$ & $1.61 \mathrm{e}$ & $1.80 f$ \\
\hline $\mathrm{SS}_{3}$ & $26.57 a b$ & $26.33 a b$ & $28.81 \mathrm{~b}$ & $3.08 a b$ & $3.13 a b$ & $3.18 d$ & $1.79 b c$ & $1.86 \mathrm{~cd}$ & $1.93 \mathrm{~cd}$ \\
\hline $\mathrm{SS}_{5}$ & $26.66 a b$ & $26.94 a b$ & $29.22 a$ & $3.15 a$ & $3.24 a$ & $3.33 a b$ & $1.93 a$ & $1.96 \mathrm{bc}$ & $2.08 \mathrm{bc}$ \\
\hline $\mathrm{PM}_{1}$ & $25.10 \mathrm{~cd}$ & $25.11 c-f$ & $25.50 \mathrm{c}$ & $2.95 b$ & $2.96 \mathrm{c}$ & 3.00de & $1.60 \mathrm{~d}$ & $1.64 \mathrm{de}$ & $2.04 \mathrm{c}$ \\
\hline $\mathrm{PM}_{2}$ & $25.33 c$ & $26.61 \mathrm{~b}$ & 29.56a & $2.98 \mathrm{~b}$ & $3.13 a b$ & $3.84 a$ & $1.73 \mathrm{c}$ & $2.21 \mathrm{a}$ & $2.85 a$ \\
\hline $\mathrm{PTR}_{1}$ & $24.20 d$ & 24.47def & 25.36de & $2.61 \mathrm{e}$ & $2.71 \mathrm{e}$ & $2.85 \mathrm{ef}$ & $1.58 \mathrm{~d}$ & $1.65 \mathrm{de}$ & $1.80 \mathrm{~d}$ \\
\hline $\mathrm{PTR}_{2}$ & $25.79 c$ & $26.07 \mathrm{~b}$ & $27.17 \mathrm{e}$ & $2.63 e$ & $2.88 d$ & $2.92 \mathrm{e}$ & $1.62 d$ & $1.69 d$ & $1.88 \mathrm{~d}$ \\
\hline$(\mathrm{SS}+\mathrm{PM})_{1}$ & $24.14 d$ & 24.29def & $24.46 \mathrm{~cd}$ & $2.77 d$ & $2.86 \mathrm{~d}$ & $2.88 \mathrm{~cd}$ & $1.59 \mathrm{~d}$ & $1.62 \mathrm{e}$ & $1.74 \mathrm{ef}$ \\
\hline$(\mathrm{SS}+\mathrm{PM})_{2}$ & $24.91 d$ & $26.26 \mathrm{~b}$ & $26.72 c$ & $2.93 \mathrm{bc}$ & $3.08 \mathrm{~b}$ & $3.09 d$ & $1.67 \mathrm{c}$ & $1.74 d$ & $1.87 d$ \\
\hline (SS+F & $27.18 \mathrm{a}$ & $27.47 a$ & $27.77 \mathrm{c}$ & $3.10 a b$ & $3.24 a$ & $3.25 \mathrm{c}$ & $1.83 b$ & $1.84 \mathrm{~cd}$ & $2.06 \mathrm{bc}$ \\
\hline RMF & $25.34 c$ & $25.81 \mathrm{c}$ & $25.72 d$ & $2.83 c$ & $2.89 \mathrm{~cd}$ & 2.98de & $1.60 \mathrm{~d}$ & 1.65de & $1.76 \mathrm{e}$ \\
\hline$F$ test $(c)$ & ns & $\mathrm{ns}$ & ns & ns & $\mathrm{ns}$ & $\mathrm{ns}$ & $\star *$ & $\star * *$ & ** \\
\hline Bio & ** & $* *$ & $\star \star *$ & $\star * *$ & $\star \star$ & ** & ** & ** & ** \\
\hline$A C x$ & $\star \star$ & ** & 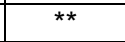 & ** & ** & ** & 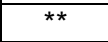 & ** & ** \\
\hline & 2.170 & 2.170 & 2.17 & 0.254 & 0.254 & 0.254 & 0.157 & 0.157 & 0.157 \\
\hline SD $1 \%$ & 2.918 & 2.918 & 2.918 & 0.342 & 0.342 & 0.342 & 0.209 & 0.209 & 0.209 \\
\hline
\end{tabular}

PM: Poultry manure, PTR: Processed town refuse.. sulphur, RMF: Recommended rates of mineral fertilizers. NS not significant, * significant, ${ }^{\star \star}$ high significant

Data in Table (7) showed that the seed yield with $\mathrm{SS}_{5}$, without added agrochemicals, $(\mathrm{SS}+\mathrm{PM})_{3}$ with gypsum and $\mathrm{PM} 2$ with sulphur was increased if compared to control by $37 ; 19$ and $16 \%$, respectively. The application of $\mathrm{SS}_{5}$ without agrochemicals, $\mathrm{PM}_{2}$ with gypsum and $\mathrm{PM}_{2}$ with sulphur produced the highest straw yield. The values were $1.93,2.21$ and $2.85 \mathrm{Mg}^{-1} \mathrm{fed}^{-1}$, respectively. These obtained results were in agreement with these of many investigators, such 
as El-Saady (1991), Fatma Osman (1998); Mohamed and Kandeel (1998). and Negm et al. (1998) .

Residual effects of remedial and amendment uses on some botanical characteristics with sunflower plants

Morphological characteristics:

Morphological characteristic of sunflower plants, i.e., Plant height, Stem diameter and Head diameter are affected by types and rates of preincorporated, recommended fertilizers and agrochemicals. The obtained data in Table (8) showed that significant differences in morphological characteristic of sunflower was recorded, the highest values were obtained by $\mathrm{PM}_{2}$ (269.50, $282.50 \cdot 290.00 \cdot 1.56,1.62,1.82,22.00,23.00$ and 23.30) of the alone, gypsum and sulphur treatments, respectively.

Dry matter leaves and stems of sunflower plant were significantly increased by previous biosolids and agrochemical application (after pea crop) at any type or rate. The positive residual effects of both $\mathrm{SS}_{5}$ and $(\mathrm{SS}+\mathrm{PM})_{3}$ treatments were always higher than PM, PTR and control treatments. These results as a harmony with these obtained by Omar and Abou Bakr (1991); Mahmoud et al. (2001); Radwan (1991) and Mohamed, (2012).

\section{Physiological characteristics:}

Plasted pigments CONTENT:

The results in Table (9) show that residual organic manures at the different types and rates with or without agrochemicals gave the highest plastid pigments content in leaves of sunflower plants compared to control. The residual effect of organic manures and agrochemicals amendments was accompanied with significant increases in total chl. content over the control. The highest values were obtained in sulphur-sewage sludge pre-treated soils at the high levels. Meanwhile, the lowest data were obtained in soil without treatments. Similar results were obtained by Abdel-Sabour(1999) and Mohamed, (2012).

2-B.2. Macronutrients content:

Data demonstrated in Table (10) show that the N, P and K contents of sunflower plants seed and straw after harvesting were highly significant as affected by types and rates of the residual organic manures and agrochemicals co-amendments as well as RMF.

Data show that the N, P and K content of different sunflower PLANTS organs were higher in $\mathrm{SS}_{5},(\mathrm{SS}+\mathrm{PM}) 3$ and $\mathrm{PM} 2$ respectively, in soil than those obtained in low rate of organic manure treatments.

Meanwhile the opposite trend was shown with high rate added of organic treated soil. On the contrast, the potassium content of different organs was lower in RMF-soil than in organic treated soil. The NPK, contents of different sunflower organs in sulphur treated soil was higher than those obtained with gypsum treated soil compared with none agrochemicals treated soil. These increases adopted the following decreasing order: Sulphur > gypsum > none. Mahmoud et al., (2001) on sunflower and Abd-Allah (1998) with sugar beet plant. 
J. Plant Production, Mansoura Univ., Vol. 5(12), December, 2014

8-9

2119 
Table (10): $\mathrm{N}, \mathrm{P}$ and $\mathrm{K}$ contents, ( $\mathrm{kg}^{-1}$ dry weight) OF sunflower plants as affected by remedial and amendments.

\begin{tabular}{|c|c|c|c|c|c|c|c|c|c|}
\hline Treatments & \multicolumn{9}{|c|}{ Added agrochemicals } \\
\hline & None & $\mathbf{G}$ & $\mathbf{S}$ & None & G & $\mathbf{S}$ & None & G & $\mathbf{S}$ \\
\hline & \multicolumn{3}{|c|}{ Seed-N } & \multicolumn{3}{|c|}{ Seed-P } & \multicolumn{3}{|c|}{ Seed-K } \\
\hline $\begin{array}{c}\text { Control } \\
\text { SS3 } \\
\text { SS5 } \\
\text { PM1 } \\
\text { PM2 } \\
\text { PTR1 } \\
\text { PTR2 } \\
\text { (SS+PM)1 } \\
\text { (SS+PM)2 } \\
\text { (SS+PM)3 } \\
\text { RMF }\end{array}$ & $\begin{array}{c}37.0 \mathrm{f} \\
39.0 \mathrm{de} \\
43.8 \mathrm{a} \\
37.3 \mathrm{f} \\
40.3 \mathrm{a} \\
38.1 \mathrm{f} \\
38.6 \mathrm{f} \\
40.7 \mathrm{c} \\
42.3 \mathrm{~b} \\
43.6 \mathrm{a} \\
43.7 \mathrm{a}\end{array}$ & $\begin{array}{c}37.5 \mathrm{~g} \\
43.5 \mathrm{c} \\
44.1 \mathrm{~b} \\
38.0 \mathrm{~g} \\
46.9 \mathrm{a} \\
40.6 \mathrm{e} \\
43.4 \mathrm{bc} \\
41.6 \mathrm{de} \\
42.8 \\
\mathrm{cde} \\
45.2 \mathrm{ab} \\
44.0 \mathrm{bc}\end{array}$ & $\begin{array}{c}40.4 \mathrm{e} \\
44.8 \mathrm{ab} \\
44.8 \mathrm{ab} \\
41.4 \mathrm{~d} \\
49.7 \mathrm{a} \\
45.3 \mathrm{~d} \\
46.4 \mathrm{bc} \\
42.6 \mathrm{c} \\
43.8 \mathrm{bc} \\
46.9 \mathrm{bc} \\
44.5 \mathrm{bc}\end{array}$ & $\begin{array}{c}7.8 \mathrm{e} \\
8.0 \mathrm{~d} \\
8.6 \mathrm{ab} \\
8.4 \mathrm{~b} \\
8.6 \mathrm{ab} \\
8.0 \mathrm{~cd} \\
8.3 \mathrm{bc} \\
8.0 \mathrm{~cd} \\
8.4 \mathrm{~b} \\
8.8 \mathrm{a} \\
8.6 \mathrm{ab}\end{array}$ & $\begin{array}{c}7.9 \mathrm{~d} \\
8.4 \mathrm{~cd} \\
8.7 \mathrm{a} \\
8.6 \mathrm{abc} \\
8.8 \mathrm{a} \\
8.3 \mathrm{a}-\mathrm{d} \\
8.5 \mathrm{ab} \\
8.2 \mathrm{bcd} \\
8.8 \mathrm{abc} \\
9.2 \mathrm{a} \\
8.8 \mathrm{a}\end{array}$ & $\begin{array}{c}8.4 c \\
8.7 c \\
8.8 b c \\
8.7 b c \\
9.0 a b \\
8.5 c \\
8.6 c \\
8.6 b c \\
9.0 a b \\
9.2 a \\
9.0 a b\end{array}$ & $\begin{array}{l}6.0 \mathrm{~d} \\
6.5 \mathrm{bc} \\
6.6 \mathrm{bc} \\
6.8 \mathrm{bc} \\
7.4 \mathrm{a} \\
6.6 \mathrm{bc} \\
7.6 \mathrm{a} \\
6.0 \mathrm{~d} \\
6.8 \mathrm{bc} \\
7.0 \mathrm{~b} \\
6.4 \mathrm{~cd}\end{array}$ & $\begin{array}{l}6.4 \mathrm{f} \\
6.8 \mathrm{ef} \\
6.9 \text { ef } \\
6.9 \text { ef } \\
7.6 \mathrm{ab} \\
7.0 \mathrm{de} \\
7.4 \mathrm{~cd} \\
7.6 \mathrm{bc} \\
8.0 \mathrm{ab} \\
8.2 \mathrm{a} \\
6.6 \mathrm{ef}\end{array}$ & $\begin{array}{c}8.0 \mathrm{bc} \\
8.2 \mathrm{ab} \\
8.6 \mathrm{a} \\
8.5 \mathrm{ab} \\
8.7 \mathrm{a} \\
8.2 \mathrm{ab} \\
8.4 \mathrm{ab} \\
7.8 \mathrm{c} \\
8.1 \mathrm{bc} \\
8.4 \mathrm{ab} \\
6.8 \mathrm{~d}\end{array}$ \\
\hline $\mathrm{F}$ test $(\mathrm{c})$ & ** & ** & *夫 & ** & ** & ** & ** & ** & ** \\
\hline & ** & ** & ** & ** & ** & ** & * & ** & ** \\
\hline & ** & $\star \star *$ & ** & ** & ** & ** & ** & ** & ** \\
\hline & 1.97 & 1.97 & 1.97 & 0.43 & 0.43 & 0.43 & 0.46 & 0.46 & 0.46 \\
\hline \multirow[t]{2}{*}{ LSD $1 \%$} & 2.68 & 2.68 & 2.68 & 0 & 0.58 & 0.58 & .62 & 0.62 & 0.62 \\
\hline & \multicolumn{3}{|c|}{ Leaves- $\mathrm{N}$} & \multicolumn{3}{|c|}{ Leaves-P } & \multicolumn{3}{|c|}{ Leaves-K } \\
\hline Control & $16.0 \mathrm{e}$ & $17.6 \mathrm{e}$ & $18.1 \mathrm{e}$ & $1.7 \mathrm{~h}$ & $2.2 \mathrm{f}$ & $2.4 \mathrm{~g}$ & $33.2 \mathrm{f}$ & $35.1 \mathrm{~g}$ & $37.6 \mathrm{~d}$ \\
\hline & 1 & & & & $2.4 \mathrm{e}$ & 2.5 ef & $\mathrm{b}$ & & $42.5 \mathrm{fg}$ \\
\hline & $21.3 \mathrm{a}$ & $21.8 \mathrm{~b}$ & $28.8 \mathrm{a}$ & $2.3 \mathrm{f}$ & $3.0 \mathrm{bc}$ & $3.1 \mathrm{~d}$ & $36.2 \mathrm{~b}$ & $43.6 \mathrm{a}$ & $55.3 \mathrm{a}$ \\
\hline PM1 & $14.2 \mathrm{e}$ & $16.6 \mathrm{~b}$ & $21.0 \mathrm{f}$ & 2.4 ef & $2.7 \mathrm{~d}$ & $3.3 \mathrm{~cd}$ & $35.2 \mathrm{de}$ & $38.2 \mathrm{~d}$ & $41.2 \mathrm{~g}$ \\
\hline & $18.7 c$ & $19.5 \mathrm{a}$ & $24.2 d$ & $2.9 \mathrm{bc}$ & $3.0 \mathrm{bc}$ & $3.3 \mathrm{~cd}$ & $39.8 \mathrm{a}$ & $47.4 \mathrm{~b}$ & $49.2 a b c$ \\
\hline PTR1 & $16.2 \mathrm{c}$ & $19.6 \mathrm{~cd}$ & $2 \mathrm{e}$ & 2.2 & $2.9 \mathrm{~b}$ & & 37. & $40.2 d$ & $41.0 \mathrm{C}$ \\
\hline & $19.9 b$ & $20.7 \mathrm{c}$ & $23.0 \mathrm{c}$ & $2.5 \mathrm{~cd}$ & $3.0 \mathrm{bc}$ & $3.4 \mathrm{fg}$ & $38.1 \mathrm{de}$ & $41.2 \mathrm{~d}$ & $43.0 \mathrm{a}$ \\
\hline$(\mathrm{SS}+\mathrm{PM}) 1$ & $17.4 \mathrm{~d}$ & $18.5 \mathrm{~d}$ & $19.9 \mathrm{~d}$ & $2.6 \mathrm{c}$ & $2.8 \mathrm{~cd}$ & $2.9 \mathrm{e}$ & $36.0 \mathrm{~cd}$ & $37.3 \mathrm{f}$ & 39.3 efg \\
\hline +PM)2 & $17.6 \mathrm{~d}$ & $19.1 \mathrm{~b}$ & $6 \mathrm{~d}$ & $2.8 \mathrm{bc}$ & $3.0 \mathrm{bc}$ & $3.1 \mathrm{~d}$ & $33.9 \mathrm{~cd}$ & $38.3 \mathrm{f}$ & 39.6 def \\
\hline (SS+ & $19.6 \mathrm{c}$ & $19.9 \mathrm{~b}$ & & & $4.3 \mathrm{a}$ & $5.2 \mathrm{a}$ & $37.9 \mathrm{~b}$ & 39.9 e & 42.2 def \\
\hline & $17.1 \mathrm{~d}$ & $18.0 \mathrm{e}$ & $18.5 \mathrm{~d}$ & $3.5 \mathrm{a}$ & $3.6 \mathrm{~b}$ & $3.7 \mathrm{~b}$ & $40.0 \mathrm{c}$ & $41.0 \mathrm{de}$ & $41.5 \mathrm{~b}$ \\
\hline $\mathrm{F}$ test $(\mathrm{c})$ & $\star *$ & ** & ** & ** & & & ** & ** & ** \\
\hline & ** & ** & ** & ** & ** & ** & ** & ** & ** \\
\hline & ** & 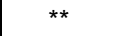 & $m$ & ** & ** & $n^{\prime}$ & $n$ & 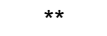 & $x$ \\
\hline & 0.5 & $0.5 \varsigma$ & & 0. & 0.26 & 0.26 & 1.11 & 1.11 & 1.11 \\
\hline LSD 1\% & 0.807 & 0.807 & 0.807 & 0.35 & 0.35 & 0.35 & 1.49 & 1.49 & 1.49 \\
\hline
\end{tabular}

SS: Sewage sludge, PM: Poultry manure, PTR: Processed town refuse, C: Control, G: gypsum, S: sulphur RMF: Recommended rates of mineral fertilizer. NS not significant * significant, ** high significant.

\section{2-B.2. Mlcronutrients content:}

Data presented in Tables (11 and 12) show that the magnitude of $\mathrm{Zn}$, $\mathrm{Mn}, \mathrm{Cu}, \mathrm{Cd}, \mathrm{Ni}$ and $\mathrm{Pb}$ in different sunflower organs (seeds and straw) cultivated in soil RMF received, as well as, those under residual organic manures treated plots were higher compared with their controls. In general, the micronutrients and heavy metals content of different sunflower organs were higher in organic treated soil than those obtained in RMF. With regard to agrochemical treated soils combined with the same organic manures and RMF, micronutrients and heavy metals of sunflower organs (seed and straw) in gypsum - treated soil were higher than those obtained in sulphur-treated soil compared with the none agrochemicals treated soils. These increases take the following decreasing order: gypsum $>$ sulphur $>$ none. 
Results in Tables (11 and 12) indicate that, values of $\mathrm{Mn}, \mathrm{Zn}$ and $\mathrm{Cu}$ in different organs had taken the decreasing orders: For $\mathrm{Zn}, \mathrm{Mn}$, Cu Seed > straw. Also, data show that, the magnitude of $\mathrm{Ni}, \mathrm{Pb}$ and $\mathrm{Cd}$ of different sunflower organs were taking the decreasing order: Straw $>$ seed.

Residual of mixed (1:1) organic manures at any level led to a decrease in the micronutrients and heavy metal contents of different sunflower organs than those obtained by the same rate of the individual organic manures.

The above-mentioned data Tables (11and 12) show clearly the beneficial effects of gypsum and sulphur on different sunflower organs quality as they increased nutrients content compared with control. Generally, under this investigation conditions, the combined of organic manures with gypsum and sulphur were more pronounced in increasing the amounts of heavy metals. But their concentrations were still low and less than the recorded.

Table (11): $\mathrm{Zn}, \mathrm{Mn}$, and Fe contents, $\left(\mathrm{mg} \mathrm{kg}^{-1}\right.$ dry weight) of sunflower plants as affected by Remedial and amendments.

\begin{tabular}{|c|c|c|c|c|c|c|c|c|c|}
\hline \multirow[t]{2}{*}{ Treatments } & \multicolumn{9}{|c|}{ Added agrochemicals } \\
\hline & None & $\mathbf{G}$ & $\mathbf{S}$ & None & $\mathbf{G}$ & $\mathbf{S}$ & None & $\mathbf{G}$ & $\mathbf{S}$ \\
\hline & \multicolumn{3}{|c|}{ Seed-Zn } & \multicolumn{3}{|c|}{ Seed-Mn } & \multicolumn{3}{|c|}{ Seed-Fe } \\
\hline Control & $32.0 \mathrm{~h}$ & $36.0 \mathrm{~g}$ & $42.0 \mathrm{f}$ & $11.0 \mathrm{~g}$ & $12.0 \mathrm{~g}$ & $13.0 \mathrm{f}$ & $47.0 \mathrm{f}$ & $55.0 \mathrm{e}$ & $58.0 \mathrm{f}$ \\
\hline SS3 & $40.0 \mathrm{de}$ & $46.0 \mathrm{~d}$ & $56.0 \mathrm{c}$ & $14.0 \mathrm{e}$ & $18.80 \mathrm{~d}$ & $19.0 d$ & $56.0 \mathrm{c}$ & $66.0 \mathrm{c}$ & $68.0 \mathrm{~d}$ \\
\hline SS5 & $54.0 \mathrm{a}$ & $62.0 \mathrm{a}$ & $66.0 \mathrm{~b}$ & $18.0 \mathrm{C}$ & $22.0 \mathrm{c}$ & $24.3 \mathrm{c}$ & $69.0 \mathrm{a}$ & $72.0 \mathrm{a}$ & $78.0 \mathrm{~b}$ \\
\hline PM1 & 38.0 ef & $48.0 \mathrm{c}$ & $50.0 \mathrm{~d}$ & $12.0 \mathrm{f}$ & $15.50 \mathrm{e}$ & $20.0 d$ & $63.0 \mathrm{~b}$ & $64.0 \mathrm{c}$ & $68.0 \mathrm{c}$ \\
\hline PM2 & $52.0 \mathrm{ab}$ & $54.0 \mathrm{bc}$ & $57.5 \mathrm{c}$ & $14.0 \mathrm{e}$ & $18.8 \mathrm{~d}$ & $24.0 \mathrm{c}$ & $68.0 \mathrm{ab}$ & $72.0 \mathrm{~b}$ & $74.0 \mathrm{bc}$ \\
\hline PTR1 & 34.0 ef & $38.0 \mathrm{fg}$ & $50.0 \mathrm{~d}$ & $12.0 \mathrm{f}$ & $14.0 \mathrm{f}$ & $16.0 \mathrm{e}$ & $55.0 \mathrm{c}$ & $65.0 \mathrm{c}$ & $72.0 \mathrm{c}$ \\
\hline PTR2 & $39.0 \mathrm{~cd}$ & $42.0 \mathrm{e}$ & $60.0 \mathrm{bc}$ & $16.0 \mathrm{~d}$ & & $24.0 \mathrm{c}$ & $68.0 \mathrm{ab}$ & $69.0 \mathrm{~b}$ & $84.0 \mathrm{a}$ \\
\hline$(S S+P M) 1$ & $35.5 \mathrm{~g}$ & $39.0 \mathrm{f}$ & $56.0 \mathrm{c}$ & $16.0 \mathrm{~d}$ & $\mathrm{~d}$ & $20.0 d$ & $53.0 \mathrm{~d}$ & $64.0 \mathrm{c}$ & $66.0 \mathrm{~d}$ \\
\hline (SS+PM)2 & $44.0 \mathrm{C}$ & $48.0 \mathrm{c}$ & $68.0 \mathrm{a}$ & $20.0 \mathrm{~b}$ & $0 \mathrm{~b}$ & $28.0 \mathrm{~b}$ & $55.0 \mathrm{c}$ & $69.0 \mathrm{~b}$ & $72.0 \mathrm{c}$ \\
\hline$(S S+P M) 3$ & $46.0 \mathrm{~b}$ & $56.0 \mathrm{~b}$ & $70.0 \mathrm{a}$ & $26.0 \mathrm{a}$ & $0 \mathrm{a}$ & $30.0 \mathrm{a}$ & $64.0 \mathrm{~b}$ & $72.0 \mathrm{a}$ & $74.0 \mathrm{bc}$ \\
\hline RMF & $36.0 \mathrm{fg}$ & 40.05 ef & $47.0 \mathrm{e}$ & $13.0 \mathrm{e}$ & $15.0 \mathrm{e}$ & $16.0 \mathrm{e}$ & $50.0 \mathrm{e}$ & $60.0 \mathrm{~d}$ & $62.0 \mathrm{e}$ \\
\hline F test $(c)$ & ** & ** & ** & ** & $\star \star$ & $\star *$ & ** & $\star \star$ & ** \\
\hline Bio (E & ** & ** & ** & ** & $\star \star$ & ** & ** & ** & ** \\
\hline$A C x$ & ** & $\star *$ & $* *$ & $* *$ & $* *$ & $\star *$ & $* *$ & $\star *$ & $* *$ \\
\hline LSD $5 \%$ & 2.384 & 2.384 & 2.384 & 1.977 & 1.977 & 1.977 & 3.346 & 3.346 & 3.346 \\
\hline \multirow[t]{2}{*}{ LSD $1 \%$} & 3.208 & 3.208 & 3.208 & 2.623 & 2.623 & 2.623 & 4.475 & 4.475 & 4.475 \\
\hline & \multicolumn{3}{|c|}{ Leave-Zn } & \multicolumn{3}{|c|}{ Leave-Mn } & \multicolumn{3}{|c|}{ Leave-Fe } \\
\hline Control & $30.0 \mathrm{f}$ & $36.0 \mathrm{~g}$ & $38.0 \mathrm{e}$ & $36.0 \mathrm{~g}$ & $40.0 \mathrm{i}$ & $46.0 \mathrm{~g}$ & $330 \mathrm{~d}$ & $364 \mathrm{f}$ & $366 \mathrm{f}$ \\
\hline SS3 & $36.0 \mathrm{~d}$ & $46.0 \mathrm{C}$ & $53.0 \mathrm{~b}$ & $64.0 \mathrm{c}$ & $72.0 \mathrm{c}$ & $76.0 \mathrm{C}$ & $352 d$ & 474 e & $572 \mathrm{c}$ \\
\hline SS5 & $47.0 \mathrm{a}$ & $53.0 \mathrm{a}$ & $58.0 \mathrm{ab}$ & $76.0 \mathrm{a}$ & $84.0 \mathrm{a}$ & $90.0 \mathrm{a}$ & $414 c$ & $524 \mathrm{~cd}$ & $644 a b$ \\
\hline PM1 & $35.0 \mathrm{~d}$ & $37.0 \mathrm{e}$ & $40.0 \mathrm{~d}$ & $39.0 \mathrm{f}$ & $46.0 \mathrm{~h}$ & $66.0 \mathrm{~d}$ & $366 \mathrm{~d}$ & 458 e & 496 de \\
\hline PM2 & $43.0 \mathrm{~b}$ & 48. $0 \mathrm{bc}$ & $50.0 \mathrm{bc}$ & $52.0 \mathrm{~d}$ & $76.0 \mathrm{~b}$ & $86.0 \mathrm{~b}$ & $446 \mathrm{bc}$ & $498 d$ & 512 de \\
\hline PTR1 & $33.0 \mathrm{e}$ & $36.0 \mathrm{f}$ & $45.0 \mathrm{c}$ & $66.0 \mathrm{bc}$ & $68.0 \mathrm{~d}$ & $86.3 b$ & $416 c$ & $561 \mathrm{bc}$ & $618 b$ \\
\hline PTR2 & $40.0 \mathrm{c}$ & $45.0 \mathrm{~cd}$ & $50.0 \mathrm{bc}$ & $69.0 \mathrm{~b}$ & $84.0 \mathrm{a}$ & $92.0 \mathrm{a}$ & $482 \mathrm{~b}$ & $655 a$ & $676 \mathrm{a}$ \\
\hline$(\mathrm{SS}+\mathrm{PM}) 1$ & 32.0 ef & $38.0 \mathrm{de}$ & $40.0 \mathrm{~d}$ & $42.0 \mathrm{e}$ & $56.0 \mathrm{e}$ & $58.0 \mathrm{e}$ & $338 d$ & 462 b & 503 e \\
\hline$(S S+P M) 2$ & $42.0 \mathrm{c}$ & $46.0 \mathrm{c}$ & $50.0 \mathrm{~b}$ & $44.0 \mathrm{e}$ & $66.0 \mathrm{de}$ & $68.0 \mathrm{~d}$ & $446 \mathrm{bc}$ & $500 \mathrm{~cd}$ & $531 d$ \\
\hline (SS+PM)3 & $48.0 \mathrm{a}$ & $50.0 \mathrm{~b}$ & $60.0 \mathrm{a}$ & $52.0 \mathrm{~d}$ & $74.0 \mathrm{~b}$ & $76.0 \mathrm{c}$ & $572 \mathrm{a}$ & 586 b & $664 \mathrm{a}$ \\
\hline RMF & $33.0 \mathrm{e}$ & $38.0 \mathrm{e}$ & $40.0 \mathrm{~d}$ & $39.0 \mathrm{f}$ & $48.0 \mathrm{~g}$ & $52.0 \mathrm{f}$ & $426 \mathrm{c}$ & $578 \mathrm{~b}$ & $636 a b$ \\
\hline F test $(c)$ & $\star \star$ & $\star *$ & $\star \star$ & $\star \star$ & $\star *$ & $\star *$ & $\star *$ & $\star *$ & $\star \star$ \\
\hline Bio (B) & ** & ** & ** & ** & ** & ** & ** & ** & ** \\
\hline$A C \times B$ & ** & ** & ** & ** & $* *$ & ** & ** & ** & $* *$ \\
\hline LSD 5\% & 3.120 & & 3.120 & 2.829 & 2.829 & 2.829 & 37.624 & 37.624 & 37.624 \\
\hline LSD $1 \%$ & 4.213 & 4.213 & 4.213 & 3.799 & 3.799 & 3.799 & 50.551 & 50.551 & 50.551 \\
\hline
\end{tabular}

SS: Sewage sludge, PM: Poultry manure, PTR: Processed town refuse, C: Control, G: gypsum, S: sulphur RMF: Recommended rates of mineral fertilizer. NS not significant * significant, ${ }^{* *}$ high significant. 


\section{B-4. Heavy metals content:}

Results in Tables (11 and 12) indicate that, values of $\mathrm{Mn}, \mathrm{Zn}$ and $\mathrm{Cu}$ in different organs had taken the decreasing orders: For $\mathrm{Zn}, \mathrm{Mn}, \mathrm{Cu}$ Seed > straw. Also, data show that, the magnitude of $\mathrm{Ni}, \mathrm{Pb}$ and $\mathrm{Cd}$ of different sunflower organs were taking the decreasing order: straw $>$ seed.

Residual of mixed (1:1) organic manures at any level led to a decrease in the micronutrients and heavy metal contents of different sunflower organs than those obtained by the same rate of the individual organic manures.

The above-mentioned data Tables (11and12) show clearly the beneficial effects of gypsum and sulphur on different sunflower organs quality as they increased nutrients content compared with control. Generally, under this investigation conditions, the combined of organic manures with gypsum and sulphur were more pronounced in increasing the amounts of heavy metals. But their concentrations were still low and less than the recorded detrimental limits which found in plants as given by Baca et al. (1995); Preer et al., (1995) ; AbdelSabour et al., (1999) and Tlustos et al. (2001) and Abari et al., in sunflower plants and Jung and Logan (1992) in sudax [Sorghum biocolo (L.)] Moench.

Data showed also that appreciable amounts of macro and micronutrients were found at lower extents. On the other hand, the contents of heavy metals in leaves and stems were higher than the seeds and still less than the toxic limits for plants.

The differences in the residual effect of biosolids may be due to their nature and chemical composition as well as the degree and rates of decomposition. However, the obtained values of $\mathrm{Fe}, \mathrm{ZN}, \mathrm{Cu}, \mathrm{Mn}, \mathrm{Ni}, \mathrm{Cd}$ and $\mathrm{Pb}$ concentrations lie within the normal levels

\section{2-C-Yield characteristics:}

Sunflower seed, Oil yield and Oil \% are affected by types and rates of pre-incorporated, recommended fertilizers and agrochemicals. The obtained data showed that significant differences in seed yield of sunflower was recorded, the highest seed yield was obtained by $\mathrm{PM}_{2}(2.56,2.90$ and 2.93 $\mathrm{Mg} \mathrm{fed}^{-1}$ ) of the alone, gypsum and sulphur treatments, respectively. The $\mathrm{PM}_{2}$ treatment surpassed that of the control by $53 \%, \mathrm{PM}_{2} 55 \%$ and $\mathrm{PM}_{2}$ with sulphur surpassed that of the control by $35 \%$.

The obtained data of seed oil percentage and contents were significantly affected by type and rate of pre-incorporated biosolids, recommended fertilizers and agrochemicals uses.

The highest seed oil yield $\left(747.52 \mathrm{~kg} \mathrm{fed}^{-1}\right)$ was obtained by application of $\mathrm{PM}_{2}$ without agrochemicals, gypsum application $821.86 \mathrm{~kg}^{-1} \mathrm{fed}^{-1}$ was obtained by $\mathrm{SS}_{5}$ and with added sulphur existed $932.32 \mathrm{~kg} \mathrm{fed}^{-1}$ was obtained by $\mathrm{PM}_{2}$. The highest percentage of oil $37.4 ; 33.8$ and $35.1 \%$ were obtained by $\mathrm{SS}_{3}$ for the alone, gypsum and sulphur treatments, respectively. It could be concluded that residual effect of both biosolids, recommended mineral fertilizes with or without agrochemicals exerts synergistic effect on oil yield obtained and oil \%,similar resuls were obtained by Abari et al., (2011) and Mohamed (2012).

The analytical results in Table (13) indicated that the highest values of seed yield/fed. were recorded with sulphur2.93 and gypsum 2.90 compared to the untreated soils (control) $2.56 \mathrm{~kg} \mathrm{fed}^{-1}$. 
J. Plant Production, Mansoura Univ., Vol. 5(12), December, 2014

12

2123 
El-Emary, F. A.A $A^{-1}$ and N.I.A. Talha ${ }^{-2}$

13

2124 
Under application of gypsum or sulphur as conditioners with or without coincorporated organic manures there was an increase in sunflower seed yield due to the improving effect on soil environment. On the other hand, the highest yield of oil was obtained from treatments amended with sulphursewage sludge at any level. Meanwhile the lowest values were recorded in the treatments without agrochemical and organic manures. These obtained results were in agreement with these of many investigators, such as Omar and Abou Bakr (1991) ; Mahmoud et al., (2001) ; Allievei et al., (1993); AbdelSabour et al., (1999); Abari et al., (2011) and Mohamed (2012).

Significant differences in yield and yield components of sunflower plants, which cultivated after pea plants. They were higher in soil treated with organic manures than those obtained from control. The above mentioned assessed parameters also showed the same trend with applied RMF. The yield and yield components of sunflower plants obtained with residual of both organic manures and gypsum or sulphur were dramatically increased over the none received agrochemicals.

\section{CONCLUSION}

Remedial and amendments, i.e, $\mathrm{SS}_{5}$, (without); PM2(with gypsum) and PM2 (with sulphur) have a significant influence on Botanical characteristics and yield of pea plants. Their residual effects extends to best botanical characteristics and yield of sunflower plants under experimental condition.

Generally, under this investigation conditions, the combined of organic manures with gypsum and sulphur were more pronounced in increasing the amounts of heavy metals. But their concentrations were still low and less than the recorded detrimental limits

\section{REFERENCES}

Abari, P.;Ghalavand, A.; Modarres, S. A. M. and M. Agha Alikhani, (2011). The effect of biofertilizers, nitrogen fertilizer and farmyard manure on yield and seed quality of sunflower (Helianthus annuus L.). J.of Agric. Tech. 7: 173-184.

Abdel-Sabour, M.F.; M.A. Abo El-Seoud and M. Rizk (1999). Physiological and chemical response of sunflower to previous organic waste composts application to sandy soils. Egypt. J. Soil Sci. 39(4): 407-420.

Abou-Bakr, M. (1988). Studies on potential benefits of solids wastes of ElAmerya District of Alexandria. The First National Conference Environmental Studies and Research. Cairo 1988. pp. 634-644.

Alina, Kabata-Pendias and Domy C. Adriano (1995). Trace Metals in Soil Amendments and Environ. Quality, p. 139-167.

Allievei, L.; A. Marchesini; C. Salardi; V. Piano and A. Ferrari (1993). Plant quality and soil residual fertility six years after a compost treatment. Bioresource Technology, 43(1): 85-89. 
Arisha, H.M. and E.A. Abd El-Bary (2000). Productivity and chemical content of spinach and pea after sulphur and sewage sludge application. Egypt J. Soil Sci. 40(4): 531-543.

Baca, M.T.; I.C. Delyado; M. De-Nobili; E. Esteban; A.J. Sanchez Raya (1995). Influence of compost maturity on nutrient status of sunflower. Communications in Soil Sci. and Plant Analysis 26(1-2): 169-181.

Chapman, H.D. and P.F. Pratt (1961). Methods of analysis for soils, plant and waters. Univ. of California, USA.

Cottenie, A.; P.M. Verloo; L. Kiekens; G. Velghe and R. Camerlynck (1982). Chemical analysis of plants and soils. Lab. Anal. and Agrochem. State Univ., Gent. Belgium. pp. 63.

Duncan, B.D. (1955). Multiple range and multiple F. test, Biometrics. 11: 142.

El-Hindi, M.H.; A.E. Sharief; S.A. El-Meursy and A.K. Seadh (2000). Response of some flax cultivars to NPK fertilizer levels. The Ninth Conference of Agronomy. September, 2-3, 2000. Agronomy Department, Faculty of Agric. Minufiya Univ.

El-Saady, S.A.A. (1991). Study on pollutants in Egyptian soils. Effects of some heavy metals on plants and soil. M.Sc. Thesis, Fac. Agric., Tanta Univ.

Fatma, Osman A.A. (1998). Effect of organic manure, phosphorus and magnesium application on yield, yield components and nutrients uptake of peas. Zagazig J. Agric. Res. 25(5): 875-888.

Gendy, E.N.; R.A. Derar and K.H.M. El-Aseel (1996). Response of soybean plants to gypsum and phosphate application. Menofiya. J. Agric. Res., 21(2): 435-441.

Gerzabak, M.H.; S.A. Mohamad; O.H. Dameberg and K. Schaffer (1992). Heavy metals in humic substance from a town refuse and a refusesewage sludge compost. (In German). Bedenkultur. 43: 21-27.

Hilal, M.H.; A.M. Selim and S.A. Korkar (1990). Response of peas to application of sulphur-urea mixtures in sandy and clay loam soils. Middle East Sulphur Symposium, Cairo, pp. 351-359.

Inskeep, W.P. and P.R. Bloom (1985): Spectroscopy in: M.F. Hipklins and N.R. Baker (eds.). Photosynthesis, Emerg. Transduction, A Plant Physiol. 77: 483-485.

Jung, J. and J.J. Logan (1992). Effects of sewage sludge cadmium concentration on chemical extractability and plant uptake. J. Environ. Qual. 12: 73-81.

Juste, C. and M. Mench (1992). Long-term application of sewage sludge and its effects an metal uptake by crops, in biogeochemistry of Trace Metals, Adriano, D.C., ED, Lewis, Publishers, Chelsea, MI, 159.

Kabata-Pendias, A. and H. Pendias (1992). Trace elements in soils and plant. $2^{\text {nd }}$ ed. CRC press, Boca. Raton. Fl.

Lindsay, W.L. and W.A. Norvell (1978). Development of a DTPA test for zinc, iron, manganese and copper. Soil Sci. Soc. Amer. J. Proc., 42: 421428.

Lorenz, O.A. and D.N. Maynard (1980). Knott's handbook for vegetable growers. Wiley and Sons, New York. 
Mahmoud, M.R.; N.M. Bader and M.H.E. Salem (2001). Influence of gypsum, sulphur and farmyard manure application on some soil properties and yield of sunflower grown on saline-sodic soil Minufiya J. Agric. Res. 26(1): 215-223.

Mashaly, S.A.; G.M. El-Sherbiny and A.M. Balba (1993). Effects of applied sewage sludge on the growth and composition of beans. Alex. Sci. Exch., 14(1): 31-47.

Mengel, K. and E.A. Kirkby (1987). Principles of plant Nutrition. International Potash Institute, Beern, Switzerland. pp. 596-600

Mohamed, F.I. and S.H. Kandeel (1998). Effect of soil sulphur application on plant growth, yield components and chemical constituents of pea cultivars. Egypt. J. Appl. Sci. 13(4): 195-209.

Mikic A,MihailovicVupina B.Dordevic V, Mhc D,Duc G,stodard F L,LejeuneHenaut I,Marget P,Hhanocq E (2011):Achievements in breeding Winter-sowen annual Legumes for temperate regions with emphasis on the cotinantal Balkans. Euphytica 180:57-67.

Negm, M.A.; R.G. Kerlous and Y.B. Besada (1998). Different sources of nitrogen and rhizobium inoculation effect on peas growing on a calcareous soil. Egypt. J. Soil Sci. 38(1-4): 69-79.

Omar, A.M. and M. Abou Baker (1991). Response of sunflower (Helianthus annuus L.) to the addition of organic matter in combination with chemical fertilizers under saline conditions. Agric. Res. Tanta Univ., 17(21): 1991-1998.

Page, A.L. (Ed). (1982). Methods of soil analysis. Part 2: Chemical and microbiological properties. (2 ${ }^{\text {nd }}$ ed.) Amer. Soc. Agron., In: Soil Sci. Soc. Amer. In, Madison, Wisconsin, USA.

Preer, J.R.; A.N. Abdi; H.S. Sekhon and G.B. Murchison (1995). Metals in urban gardens effect of lime and sludge. J. of Environ. Science and Health 30(a): 2041-2056.

Radwan, F. (1991). Effect of sewage sludge on some sunflower and barley characteristics. Annals of Agricultural Science. Moshtohor, 29(4): 13331344.

Raven, K.P. and R.H. Loeppert (1997). Trace element composition of fertilizers and soil amendments. J. Environ. Qual. 26: 551-557.

Snell, F.D. and C.T. Snell (1977). Calorimetric methods of analysis. D. Van Nostrand Company, Inc.: 551-552

Tlustos, P.; D. Pavilikova; J. Balik; J. Szakova and A. Hanc (2001). The availability of sewage sludge derived cadmium and nickel by crops planted on soils of different types. Soils and Fertilizer 64(5): 820 
اثر الاستخدامات ألعلاجيه و المحسنات علي بعض الخصائص النباتيه و المحصول

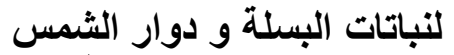

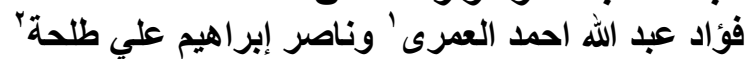

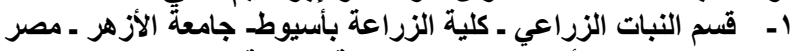

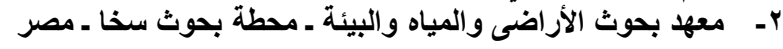

أجريت هذه الدراسة تحت الظروف الحقلية لمحطه بحوث سخا بمحافظة كفر الثيخ فى موسمين

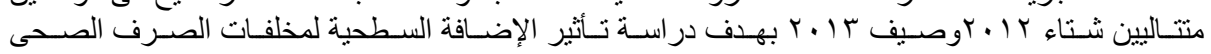
، سماد الدواجن Sewage sludge (SS)

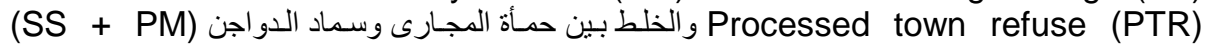

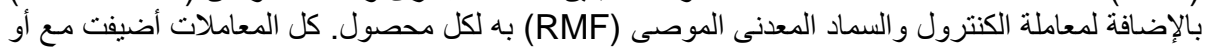

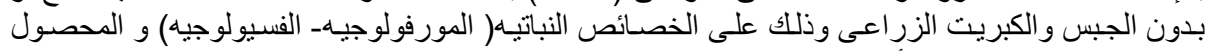

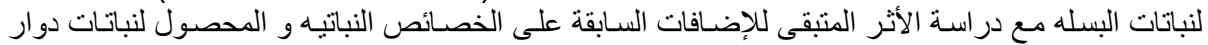

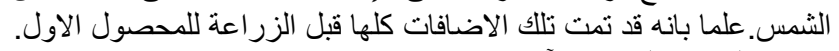

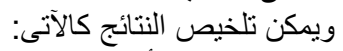

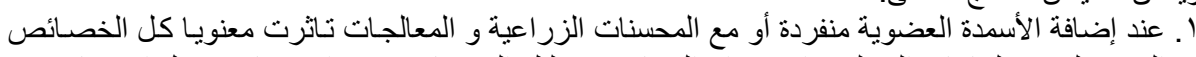

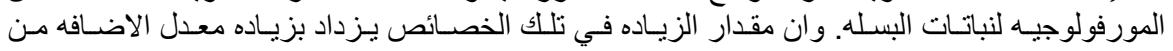

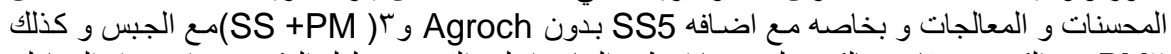
مع الكبريت مقارنه بالكتنرول. بينما اعطت النتائج اعلي القيم مع دليل البذور عند استخدام المعامله

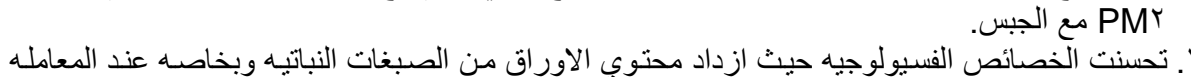

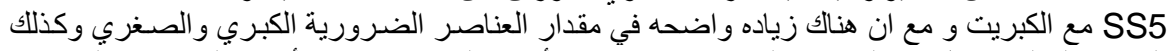

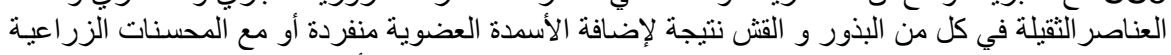

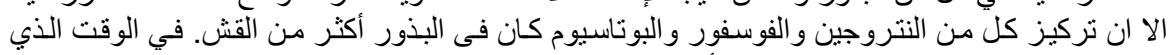

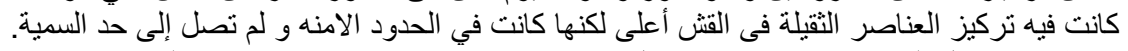

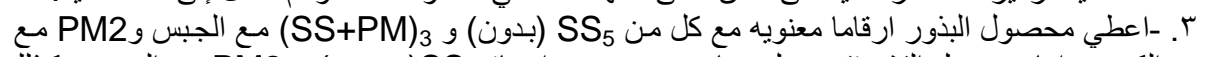

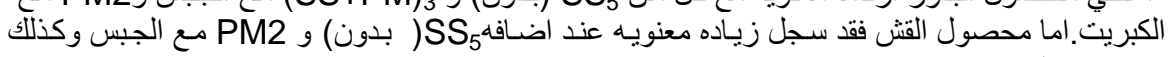

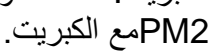

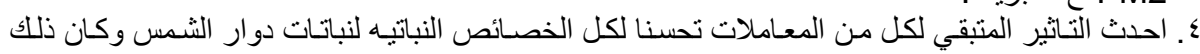

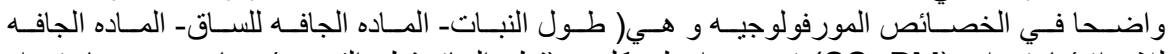
للاور اق)باستخدام

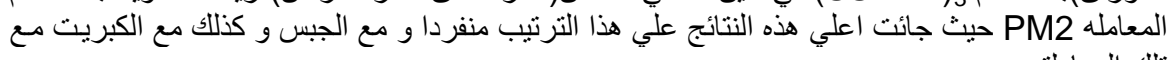

تانلك المعاملتين.

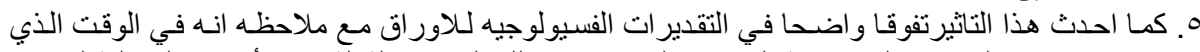

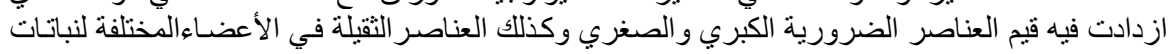

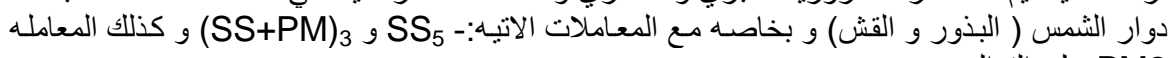
PM2

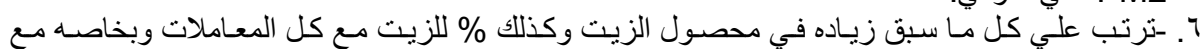

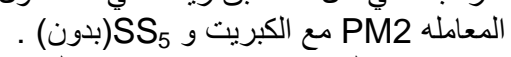

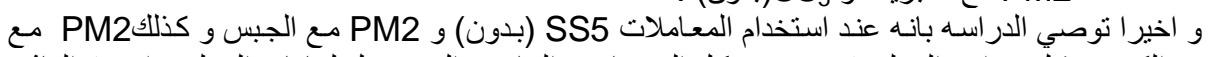

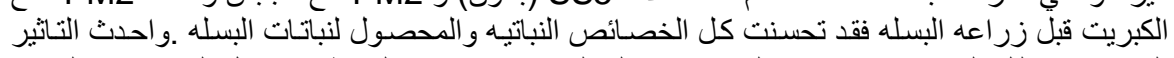

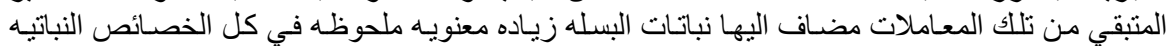

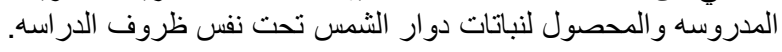


J. Plant Production, Mansoura Univ., Vol. 5(12), December, 2014

2129 
J. Plant Production, Mansoura Univ., Vol. 5 (12): 2105- 2128, 2014

Table (8): Morphological characteristic of sunflower plants as affected by remedial and amendments

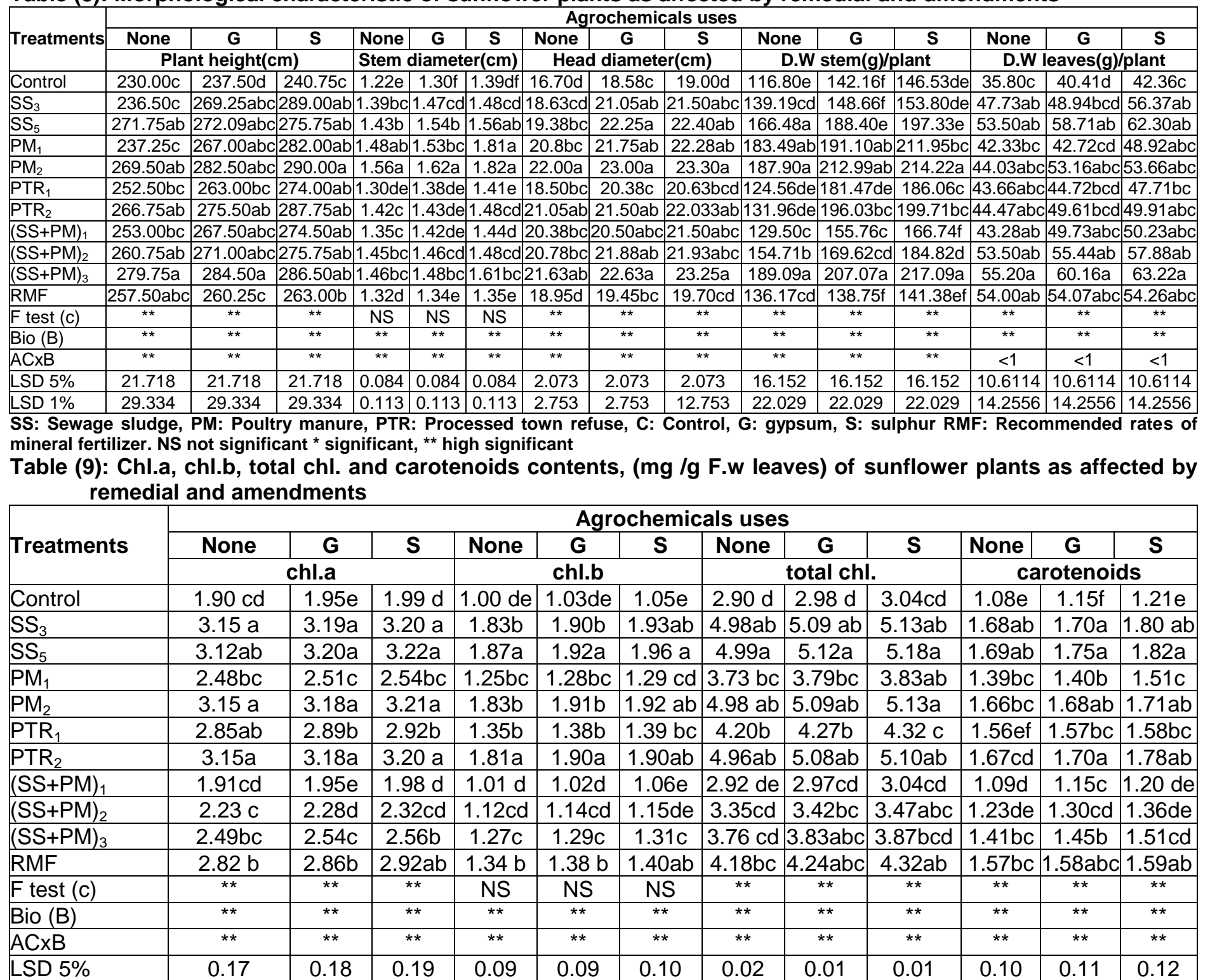

SS: Sewage sludge PM: Poultry manure, PTR: Processed town refuse, C: Control, G: gypsum, S: sulphur RMF: Recommended rates of minera

fertilizer. NS not significant ${ }^{*}$ significant, ${ }^{* *}$ high significant 
J. Plant Production, Mansoura Univ., Vol. 5(12), December, 2014

Table (2):Morphological characteristics of pea plants as affected by remedial and amendments treatments

\begin{tabular}{|c|c|c|c|c|c|c|c|c|c|}
\hline \multirow{3}{*}{ Treatments } & \multicolumn{9}{|c|}{ Agrochemicals uses } \\
\hline & None & $\mathbf{G}$ & $\mathbf{S}$ & None & $\mathbf{G}$ & $\mathbf{S}$ & None & $\mathbf{G}$ & $\mathbf{S}$ \\
\hline & \multicolumn{3}{|c|}{ D. W (g) / plant } & \multicolumn{3}{|c|}{ Seed index Weight 100(g) } & \multicolumn{3}{|c|}{ Protein \% } \\
\hline Control & $13.30 \mathrm{bc}$ & $13.62 \mathrm{bc}$ & $13.81 \mathrm{c}$ & $25.37 \mathrm{f}$ & $25.46 \mathrm{~g}$ & $27.70 \mathrm{e}$ & $24.16 \mathrm{e}$ & $25.44 a b$ & $26.63 b c$ \\
\hline $\mathrm{SS}_{3}$ & 13.38ab & $14.47 \mathrm{c}$ & $15.25 b$ & $28.95 \mathrm{~cd}$ & $29.83 d$ & $32.95 b$ & $24.94 a$ & $25.52 d$ & $26.46 a$ \\
\hline $\mathrm{SS}_{5}$ & $15.00 \mathrm{a}$ & $15.50 \mathrm{bc}$ & $15.76 \mathrm{bc}$ & $29.04 c$ & $31.53 c$ & $32.39 b$ & $26.32 a$ & $27.96 \mathrm{bcd}$ & $28.72 b-e$ \\
\hline $\mathrm{PM}_{1}$ & 13.60abc & $13.73 b$ & $13.97 \mathrm{c}$ & $26.12 \mathrm{e}$ & $26.91 f$ & $31.65 b$ & $26.06 \mathrm{cde}$ & $27.92 a b$ & $28.66 \mathrm{~b}-\mathrm{e}$ \\
\hline $\mathrm{PM}_{2}$ & 14.49abc & $18.20 \mathrm{a}$ & $23.75 a$ & $27.80 \mathrm{e}$ & $28.84 \mathrm{e}$ & $34.92 a$ & $27.05 \mathrm{bcd}$ & $28.27 a$ & $30.00 \mathrm{bcd}$ \\
\hline PTR $_{1}$ & 13.11abc & $13.17 \mathrm{c}$ & $14.87 \mathrm{bc}$ & $28.07 \mathrm{~cd}$ & $29.51 d$ & $29.90 d$ & $24.85 \mathrm{de}$ & $25.14 d$ & $25.83 e$ \\
\hline $\mathrm{PTR}_{2}$ & $13.47 c$ & $13.55 c$ & $15.15 b c$ & $31.30 \mathrm{ab}$ & $30.37 \mathrm{~cd}$ & $30.90 c$ & 26.24cde & 26.24abc & 26.91cde \\
\hline$(\mathrm{SS}+\mathrm{PM})_{1}$ & $12.93 c$ & $13.50 \mathrm{c}$ & $13.60 \mathrm{c}$ & $27.46 \mathrm{~d}$ & $30.49 \mathrm{~cd}$ & $31.16 c$ & $25.27 a b c$ & $26.61 \mathrm{~cd}$ & 26.93de \\
\hline$(\mathrm{SS}+\mathrm{PM})_{2}$ & 13.86abc & $13.89 \mathrm{bc}$ & $13.98 c$ & $30.29 b$ & $31.33 c$ & $32.98 b$ & 26.94b-e & $27.12 a$ & $27.65 b-e$ \\
\hline$(\mathrm{SS}+\mathrm{PM})_{3}$ & $15.00 \mathrm{ab}$ & $15.45 b c$ & $15.83 \mathrm{bc}$ & $31.02 a b$ & $33.08 a$ & $33.81 \mathrm{ab}$ & $27.55 a-d$ & $28.46 a$ & $29.02 b-e$ \\
\hline RMF & $13.58 \mathrm{abc}$ & $14.08 \mathrm{bc}$ & $14.99 b c$ & $32.57 a$ & $32.70 b$ & $32.95 b$ & $29.47 a b$ & 29.69ab & $29.86 b$ \\
\hline F test (c) & * & * & * & * & * & * & * & * & * \\
\hline Bio (B) & ** & ** & ** & ** & ** & ** & $\star \star$ & ** & ** \\
\hline $\mathrm{ACxB}$ & ** & ** & $* *$ & ** & ** & ** & ** & ** & ** \\
\hline LSD 5\% & 2.630 & 2.630 & 2.630 & 2.001 & 2.114 & 2.114 & 2.501 & 2.501 & 2.501 \\
\hline LSD 1\% & 2.756 & 2.756 & 2.756 & 2.830 & 2.830 & 2.830 & 3.343 & 3.343 & 3.343 \\
\hline
\end{tabular}

SS: Sewage sludge. PM: Poultry manure, PTR: Processed town refuse.. G: gypsum, S: sulphur, RMF: Recommended rates of mineral fertilizers. NS not significant, * significant, ${ }^{* *}$ high significant 
El-Emary, F. A.A ${ }^{-1}$ and N.I.A. Talha ${ }^{-2}$

Table (3): Chl.a , chl.b, total chl. and carotenoids contents, (mg /g F.w leavs) OF PEA plants as affected by remedial and amendmens

\begin{tabular}{|c|c|c|c|c|c|c|c|c|c|c|c|c|}
\hline \multirow{3}{*}{ Treatments } & \multicolumn{12}{|c|}{ Agrochemicals uses } \\
\hline & None & G & $\mathbf{S}$ & None & $\mathbf{G}$ & $\mathbf{S}$ & None & G & $\mathbf{S}$ & None & G & $\mathbf{S}$ \\
\hline & \multicolumn{3}{|c|}{ chl.a } & \multicolumn{3}{|c|}{ chl.b } & \multicolumn{3}{|c|}{ total chl. } & \multicolumn{3}{|c|}{ carotenoids } \\
\hline Control & $2.33 f$ & $2.38 f$ & $2.44 \mathrm{f}$ & $2.07 \mathrm{~g}$ & $2.11 \mathrm{~g}$ & $2.15 \mathrm{~g}$ & $4.40 \mathrm{~g}$ & $4.49 \mathrm{~g}$ & $5.59 \mathrm{~g}$ & $1.01 \mathrm{f}$ & $1.02 f$ & $1.03 \mathrm{e}$ \\
\hline $\mathrm{SS}_{3}$ & $3.78 b$ & $3.84 b$ & $3.86 b$ & $3.26 b$ & $3.26 b$ & $3.27 b$ & $7.04 b$ & $7.10 \mathrm{~b}$ & $7.13 b$ & $1.18 b$ & $1.21 \mathrm{~b}$ & $1.23 b$ \\
\hline $\mathrm{SS}_{5}$ & $4.34 a$ & $4.29 a$ & $4.24 a$ & $3.31 a$ & $3.35 a$ & $3.39 a$ & $7.35 a$ & $7.64 a$ & $7.63 a$ & $1.37 a$ & $1.42 \mathrm{a}$ & $1.47 \mathrm{a}$ \\
\hline $\mathrm{PM}_{1}$ & $3.20 d$ & $3.21 d$ & $3.22 d$ & $2.87 d$ & $2.85 d$ & $2.82 d$ & $6.07 e$ & $6.06 \mathrm{e}$ & $6.04 \mathrm{e}$ & $1.11 \mathrm{~d}$ & $1.11 \mathrm{de}$ & $1.12 \mathrm{e}$ \\
\hline $\mathrm{PM}_{2}$ & $3.70 b$ & $3.73 b$ & $3.77 \mathrm{~b}$ & $3.10 \mathrm{~b}$ & $3.13 b$ & $3.16 \mathrm{~b}$ & $6.80 \mathrm{~b}$ & $6.86 b$ & $6.93 b$ & $1.13 b$ & $1.16 \mathrm{~b}$ & $1.18 b$ \\
\hline $\mathrm{PTR}_{1}$ & $2.69 \mathrm{e}$ & $2.80 \mathrm{e}$ & $2.91 \mathrm{e}$ & $2.31 \mathrm{e}$ & $2.40 \mathrm{e}$ & $2.49 \mathrm{e}$ & $5.00 f$ & $5.20 f$ & $5.40 f$ & $1.07 e$ & 1.08ef & $1.09 f$ \\
\hline $\mathrm{PTR}_{2}$ & $2.69 \mathrm{e}$ & $2.78 f$ & $2.78 f$ & $2.15 f$ & $2.27 f$ & $2.38 f$ & $4.84 \mathrm{~g}$ & $5.05 \mathrm{~g}$ & $5.16 \mathrm{~g}$ & $1.06 \mathrm{e}$ & $1.08 \mathrm{ef}$ & $1.09 f$ \\
\hline$(\mathrm{SS}+\mathrm{PM})_{1}$ & $2.58 \mathrm{e}$ & $2.64 \mathrm{e}$ & $2.66 \mathrm{e}$ & $2.24 f$ & $2.27 f$ & $2.30 f$ & $4.82 f$ & $4.91 \mathrm{f}$ & $4.96 f$ & $1.05 e$ & $1.07 \mathrm{ed}$ & $1.09 \mathrm{~d}$ \\
\hline$(\mathrm{SS}+\mathrm{PM})_{2}$ & $3.11 d$ & $3.14 d$ & $3.17 d$ & $2.75 e$ & $2.76 \mathrm{e}$ & $2.77 e$ & $5.85 e$ & $5.90 e$ & $5.94 e$ & $1.07 \mathrm{~d}$ & $1.09 \mathrm{~d}$ & $1.10 \mathrm{~d}$ \\
\hline$(\mathrm{SS}+\mathrm{PM})_{3}$ & $3.77 \mathrm{~b}$ & $3.78 \mathrm{~b}$ & $3.79 b c$ & $3.25 b$ & 3.30ab & $3.35 a$ & $7.02 \mathrm{c}$ & $7.08 \mathrm{c}$ & $7.14 \mathrm{c}$ & $1.13 c$ & $1.13 \mathrm{c}$ & $1.14 \mathrm{~d}$ \\
\hline RMF & $3.66 \mathrm{c}$ & $3.69 c$ & $3.71 \mathrm{c}$ & $3.06 \mathrm{c}$ & $3.13 c$ & $3.19 \mathrm{c}$ & $6.72 d$ & $6.82 d$ & $6.90 \mathrm{~d}$ & $1.14 \mathrm{c}$ & $1.16 \mathrm{c}$ & $1.17 \mathrm{c}$ \\
\hline F test $(\mathrm{c})$ & ${ }^{\star *}$ & $\star \star$ & $\star \star$ & NS & NS & NS & ${ }^{\star *}$ & $\star \star$ & $* *$ & $\star \star$ & $* *$ & $\star \star *$ \\
\hline Bio (B) & ** & ** & ** & ** & ** & $\star * *$ & ** & ** & ** & ** & ** & ** \\
\hline $\mathrm{ACXB}$ & ** & ** & ** & ** & $\star \star *$ & ** & ** & ** & ** & ** & ** & ** \\
\hline LSD 5\% & 0.36 & 0.26 & 0.22 & 0.08 & 0.16 & 0.15 & 0.42 & 0.42 & 0.43 & 0.06 & 0.05 & 0.06 \\
\hline
\end{tabular}

SS: Sewage sludge, PM: Poultry manure, PTR: Processed town refuse, C: Control, G: gypsum, S: sulphur RMF: Recommended rates of mineral fertilizer.

NS not significant ${ }^{*}$ significant, ${ }^{* *}$ high significant 
Table (4): N, P and K contents, ( $\mathrm{g} \mathrm{kg}^{-1}$ dry weight) of pea plants as affected by remedial and amendments

\begin{tabular}{|c|c|c|c|c|c|c|c|c|c|}
\hline \multirow[t]{3}{*}{ Treatments } & \multicolumn{9}{|c|}{ Added agrochemicals } \\
\hline & None & G & $\mathbf{S}$ & None & $\mathrm{G}$ & S & None & G & $\mathbf{S}$ \\
\hline & \multicolumn{3}{|c|}{ Seed-N } & \multicolumn{3}{|c|}{ Seed-P } & \multicolumn{3}{|c|}{ Seed-K } \\
\hline Control & $38.65 \mathrm{e}$ & $39.10 \mathrm{ab}$ & $40.40 \mathrm{ab}$ & $4.30 \mathrm{c}$ & $4.90 \mathrm{abc}$ & $4.75 \mathrm{ab}$ & $9.45 \mathrm{~d}$ & $10.43 \mathrm{c}$ & $9.83 \mathrm{c}$ \\
\hline $\begin{array}{l}\text { SS3 } \\
\text { SS5 }\end{array}$ & $41.90 \mathrm{ab}$ & $45.72 \mathrm{~cd}$ & $47.95 \mathrm{bc}$ & $4.80 \mathrm{abc}$ & $4.83 \mathrm{bc}$ & $5.00 \mathrm{a}$ & $10.40 \mathrm{bcd}$ & $11.18 \mathrm{c}$ & $10.60 \mathrm{abc}$ \\
\hline & $\begin{array}{l}43.50 \mathrm{a} \\
41.70 \mathrm{de}\end{array}$ & $\begin{array}{l}48.13 \mathrm{bcd} \\
45.28 \mathrm{abe}\end{array}$ & $\begin{array}{l}51.13 \mathrm{a} \\
46.85 \mathrm{bc}\end{array}$ & $\begin{array}{l}5.00 \mathrm{a} \\
4.40 \mathrm{bc}\end{array}$ & $\begin{array}{r}5.43 \mathrm{a} \\
5.08 \mathrm{ab}\end{array}$ & $\begin{array}{l}5.00 \mathrm{a} \\
4.60 \mathrm{ab}\end{array}$ & $\begin{array}{r}10.68 \mathrm{a} \\
10.50 \mathrm{bcd}\end{array}$ & $\begin{array}{l}12.30 \mathrm{c} \\
11.64 \mathrm{bc}\end{array}$ & $\begin{array}{l}11.45 \mathrm{ab} \\
11.15 \mathrm{abc}\end{array}$ \\
\hline PM2 & $43.28 \mathrm{~b}-\mathrm{e}$ & $46.42 \mathrm{a}$ & $48.08 \mathrm{bc}$ & $4.80 \mathrm{abc}$ & $5.10 \mathrm{ab}$ & $4.85 \mathrm{ab}$ & $11.23 \mathrm{ab}$ & $13.08 \mathrm{a}$ & $11.65 \mathrm{ab}$ \\
\hline $\begin{array}{l}\text { PTR1 } \\
\text { PTR2 }\end{array}$ & $40.98 \mathrm{cde}$ & $\begin{array}{l}41.23 d \\
43.18\end{array}$ & $\begin{array}{l}42.33 \mathrm{c} \\
4505 \mathrm{co}\end{array}$ & $4.43 \mathrm{abc}$ & $4.65 \mathrm{bc}$ & $4.54 \mathrm{~b}$ & $\begin{array}{l}9.77 \mathrm{~cd} \\
10.10 \mathrm{cc}\end{array}$ & $10.70 \mathrm{c}$ & $10.30 \mathrm{bc}$ \\
\hline$(\mathrm{SS}+\mathrm{PM}) 1$ & $\begin{array}{l}42.25 \mathrm{cde} \\
40.10 \mathrm{~b}-\mathrm{e}\end{array}$ & $\begin{array}{l}\begin{array}{l}43.18 \mathrm{ad} \\
41.98 \mathrm{~d}\end{array}\end{array}$ & $\begin{array}{l}4.05 \mathrm{bc} \\
43.80 \mathrm{c}\end{array}$ & $\begin{array}{l}4.90 \mathrm{ab} \\
4.48 \mathrm{abc}\end{array}$ & $\begin{array}{l}5.00 \mathrm{ab} \\
4.88 \mathrm{c}\end{array}$ & $\begin{array}{l}4.55 \mathrm{ab} \\
4.55 \mathrm{ab}\end{array}$ & $\begin{array}{l}10.10 \mathrm{bcd} \\
10.50 \mathrm{abc}\end{array}$ & $\begin{array}{l}11.45 \mathrm{bc} \\
11.85 \mathrm{bc}\end{array}$ & $\begin{array}{l}10.60 \mathrm{bc} \\
10.85 \mathrm{bc}\end{array}$ \\
\hline$(\mathrm{SS}+\mathrm{PM}) 2$ & $44.08 \mathrm{a}-\mathrm{d}$ & $44.80 \mathrm{ab}$ & $47.22 \mathrm{bc}$ & $4.53 \mathrm{abc}$ & $4.98 \mathrm{bc}$ & $4.78 \mathrm{ab}$ & $11.25 \mathrm{ab}$ & $12.00 \mathrm{bc}$ & $11.59 \mathrm{bc}$ \\
\hline $\begin{array}{l}(\mathrm{SS}+\mathrm{PM}) 3 \\
\mathrm{BMF}\end{array}$ & $43.23 \mathrm{a}-\mathrm{d}$ & $45.73 \mathrm{a}$ & $48.23 \mathrm{bc}$ & $4.68 \mathrm{abc}$ & $5.00 \mathrm{bc}$ & $4.88 \mathrm{ab}$ & $11.45 \mathrm{ab}$ & $12.10 \mathrm{ab}$ & $11.78 \mathrm{a}$ \\
\hline \multirow{5}{*}{$\begin{array}{l}\text { F test (c) } \\
\text { Bio (B) } \\
\text { AC X B } \\
\text { LSD 5\% } \\
\text { LSD 1\% } \\
\end{array}$} & & & & & & $5.05 \mathrm{a}$ & ns & $\frac{11.58 \mathrm{DC}}{\mathrm{ns}}$ & ns \\
\hline & $\stackrel{* *}{\star *}$ & ${ }_{* \star}^{* \star}$ & ** $* *$ & ${ }^{* *}$ & $* *$ & $* *$ & 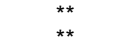 & ** & ** \\
\hline & 4.41 & 4.41 & 4.41 & ns & $\begin{array}{c}\text { ns } \\
049\end{array}$ & ns & 128 & 128 & 128 \\
\hline & $\begin{array}{l}4.41 \\
588\end{array}$ & $\begin{array}{l}4.41 \\
5.88\end{array}$ & $\begin{array}{l}4.41 \\
588\end{array}$ & 0.49 & 0.49 & 0.49 & 1.28 & 1.28 & 1.28 \\
\hline & \multicolumn{3}{|c|}{ Straw $-\mathrm{N}$} & \multirow{2}{*}{\multicolumn{3}{|c|}{ Straw-P }} & \multicolumn{3}{|c|}{$\frac{1.73}{\text { Straw-K }}$} \\
\hline Control & $16.60 \mathrm{f}$ & 17.75 & 20.65 ef & $1.10 \mathrm{~d}$ & & & $5.90 \mathrm{e}$ & $6.78 \mathrm{f}$ & $8.48 \mathrm{~d}$ \\
\hline & $17.23 \mathrm{~b}$ & $17.60 \mathrm{c}$ & $22.10 \mathrm{f}$ & $1.35 \mathrm{bc}$ & $1.50 \mathrm{~cd}$ & $1.40 \mathrm{~d}$ & $7.80 \mathrm{~cd}$ & $7.98 \mathrm{e}$ & $8.80 \mathrm{~cd}$ \\
\hline & $\begin{array}{l}17.63 \mathrm{a} \\
17.30 \mathrm{of}\end{array}$ & $\begin{array}{l}20.00 \mathrm{c} \\
1800 \mathrm{c}\end{array}$ & $26.20 \mathrm{a}-\mathrm{d}$ & $1.38 \mathrm{ab}$ & $1.65 \mathrm{~cd}$ & $1.45 \mathrm{~cd}$ & $\begin{array}{l}8.70 \mathrm{a} \\
805\end{array}$ & $11.25 \mathrm{cde}$ & $\begin{array}{l}11.70 \mathrm{a} \\
10.50 \mathrm{~b}\end{array}$ \\
\hline PM2 & 18.00 def & $18.63 \mathrm{abc}$ & $\begin{array}{l}18.48 \mathrm{C}-1 \\
19.05 \mathrm{c}-\mathrm{f}\end{array}$ & $1.35 \mathrm{a}$ & $1.90 \mathrm{~cd}$ & $\begin{array}{l}1.48 \mathrm{de} \\
1.65\end{array}$ & $\begin{array}{l}8.05 \mathrm{ca} \\
8.40 \mathrm{c}\end{array}$ & $11.10 \mathrm{a}$ & $\begin{array}{l}11.75 \mathrm{a} \\
1.75\end{array}$ \\
\hline |PTR1 & $18.18 \mathrm{cde}$ & $18.63 \mathrm{abc}$ & $19.33 \mathrm{def}$ & $1.45 \mathrm{bc}$ & $1.58 \mathrm{bcd}$ & $\begin{array}{l}1.50 \mathrm{c} \\
158 \mathrm{~h}\end{array}$ & $7.40 \mathrm{~cd}$ & $7.90 \mathrm{e}$ & $8.20 \mathrm{~d}$ \\
\hline $\begin{array}{l}\text { PTR2 } \\
\text { (SS+PM)1 }\end{array}$ & $\begin{array}{l}19.03 \mathrm{bc} \\
19.70 \mathrm{~cd}\end{array}$ & $\begin{array}{l}20.23 \mathrm{a} \\
2000 \mathrm{ab}\end{array}$ & 20.30 b- $f$ & $\begin{array}{l}1.48 \mathrm{bc} \\
1.33 \mathrm{~cd}\end{array}$ & $\begin{array}{l}1.63 \mathrm{abc} \\
148 \mathrm{bcd}\end{array}$ & $\begin{array}{l}1.580 \\
138 d\end{array}$ & $\begin{array}{l}7.80 \mathrm{~cd} \\
810 \mathrm{~cd}\end{array}$ & $\begin{array}{l}8.30 \mathrm{de} \\
880 \mathrm{cde}\end{array}$ & $8.80 \mathrm{~d}$ \\
\hline$(\mathrm{SS}+\mathrm{PM}) 2$ & 20.03 bc & $20.28 \mathrm{a}$ & $20.80 \mathrm{ab}$ & $\begin{array}{l}1.43 \mathrm{bc} \\
\text { - }\end{array}$ & $1.53 \mathrm{abc}$ & $\begin{array}{l}1.50 \mathrm{c} \\
\end{array}$ & $9.20 \mathrm{~b}$ & $9.58 \mathrm{~cd}$ & $10.00 \mathrm{~b}$ \\
\hline$(\mathrm{SS}+\mathrm{PM}) 3$ & $20.63 \mathrm{bc}$ & $20.60 \mathrm{a}$ & $21.83 \mathrm{a}$ & $1.58 \mathrm{a}$ & $1.88 \mathrm{a}$ & $1.68 \mathrm{~b}$ & $9.60 \mathrm{~b}$ & $10.05 \mathrm{bc}$ & $10.50 \mathrm{ab}$ \\
\hline F test (c) & ns & Ns & $\frac{19.48 \mathrm{~b}-\mathrm{e}}{\mathrm{ns}}$ & $\frac{1.68 \mathrm{ab}}{<1}$ & $\frac{1.88 \mathrm{a}}{<1}$ & $1.18 \mathrm{a}$ & & $7.98 \mathrm{e}$ & \\
\hline Bio (B) & ${ }^{\star \star *}$ & 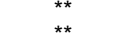 & $* *$ & $* *$ & 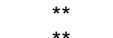 & ** & $* *$ & $* *$ & ** \\
\hline LSD $5 \%$ & 2.11 & 2.11 & $211+2-1$ & 029 & 039 & 029 & 092 & 092 & 0.92 \\
\hline LSD $1 \%$ & 2.90 & 2.90 & 2.90 & $\begin{array}{l}.29 \\
0.39\end{array}$ & 0.39 & 0.39 & 1.23 & 1.23 & 1.23 \\
\hline
\end{tabular}

SS: Sewage sludge, PM: Poultry manure, PTR: Processed town refuse, C: Control, G: gypsum. S: sulphur, RMF: Recommended rates of mineral fertilizer. NS not significant ${ }^{*}$ significant, ${ }^{* \star}$ high significant 
El-Emary, F. A.A $A^{-1}$ and N.I.A. Talha ${ }^{-2}$

Table (6): $\mathrm{Cd}, \mathrm{Cu}, \mathrm{Ni}$ and $\mathrm{Pb}$ contents, $\left(\mathrm{mg} \mathrm{kg}^{-1}\right.$ dry weight) of pea plants as affected by Remedial and amendments. Treatments

\begin{tabular}{|c|c|c|c|c|c|c|c|c|c|c|c|c|}
\hline \multirow[t]{2}{*}{ I reatments } & \multicolumn{12}{|c|}{ Added agrochemicals } \\
\hline & None & G & $\mathbf{S}$ & None & G & & None & G & $\mathbf{S}$ & None & $\mathbf{G}$ & $\mathbf{S}$ \\
\hline $\begin{array}{l}\text { Control } \\
\text { SS3 } \\
\text { SS5 } \\
\text { PM1 } \\
\text { PM2 } \\
\text { PIR1 } \\
\text { PTR2 } \\
\text { PTS+PM)1 } \\
\text { (SS+PM) } \\
\text { (SS+PM) } 2 \\
\text { SS+PM)3 } \\
\text { RMF } \\
\end{array}$ & $\begin{array}{l}0.02 \mathrm{f} \\
0.04 \mathrm{~d} \\
0.10 \mathrm{a} \\
0.04 \mathrm{~d} \\
0.06 \mathrm{~cd} \\
0.06 \mathrm{~cd} \\
0.08 \mathrm{bc} \\
0.06 \mathrm{~cd} \\
0.08 \mathrm{bc} \\
0.10 \mathrm{a} \\
0.03 \mathrm{e}\end{array}$ & $\begin{array}{l}\text { Seed-c } \\
0.03 \mathrm{f} \\
0.10 \mathrm{bc} \\
0.12 \mathrm{~b} \\
0.08 \mathrm{~cd} \\
0.10 \mathrm{bc} \\
0.08 \mathrm{~cd} \\
0.10 \mathrm{bc} \\
0.07 \mathrm{~d} \\
0.10 \mathrm{bc} \\
0.13 \mathrm{a} \\
0.04 \mathrm{e}\end{array}$ & $\begin{array}{l}0.04 \mathrm{~g} \\
0.11 \mathrm{~cd} \\
0.18 \mathrm{a} \\
0.09 \mathrm{e} \\
0.12 \mathrm{c} \\
0.09 \mathrm{e} \\
0.11 \mathrm{~cd} \\
0.08 \mathrm{~cd} \\
0.11 \mathrm{~cd} \\
0.14 \mathrm{~b} \\
0.05 \mathrm{f} \\
\end{array}$ & $\begin{array}{l}3.0 \mathrm{f} \\
3.4 \mathrm{ef} \\
3.5 \mathrm{~d} \\
3.2 \mathrm{f} \\
3.4 \mathrm{ef} \\
5.5 \mathrm{c} \\
6.0 \mathrm{~b} \\
3.5 \mathrm{e} \\
4.5 \mathrm{~d} \\
8.0 \mathrm{a} \\
3.5 \mathrm{e} \\
\end{array}$ & $\begin{array}{l}\text { Seed-Cu } \\
3.2 \mathrm{f} \\
3.5 \mathrm{ef} \\
4.0 \mathrm{~cd} \\
3.3 \mathrm{f} \\
3.5 \mathrm{ef} \\
6.0 \mathrm{~cd} \\
8.0 \mathrm{~b} \\
3.8 \mathrm{~d} \\
5.5 \mathrm{~d} \\
9.0 \mathrm{a} \\
4.0 \mathrm{e} \\
\end{array}$ & $\begin{array}{l}3.4 \mathrm{e} \\
3.6 \mathrm{e} \\
4.3 \mathrm{e} \\
3.4 \mathrm{e} \\
4.3 \mathrm{f} \\
8.0 \mathrm{c} \\
9.0 \mathrm{~b} \\
5.0 \mathrm{de} \\
9.0 \mathrm{~b} \\
10.0 \mathrm{a} \\
5.0 \mathrm{de}\end{array}$ & $\begin{array}{l}0.30 \mathrm{i} \\
0.42 \mathrm{f} \\
0.50 \mathrm{~d} \\
0.46 \mathrm{ef} \\
0.56 \mathrm{~d} \\
0.64 \mathrm{~b} \\
0.66 \mathrm{a} \\
0.38 \mathrm{~g} \\
0.50 \mathrm{e} \\
0.62 \mathrm{c} \\
0.44 \mathrm{ef}\end{array}$ & $\begin{array}{l}0.38 \mathrm{ef} \\
0.44 \mathrm{ef} \\
0.56 \mathrm{ef} \\
0.48 \mathrm{f} \\
0.58 \mathrm{de} \\
0.66 \mathrm{~cd} \\
0.67 \mathrm{bc} \\
0.54 \mathrm{f} \\
0.56 \mathrm{ef} \\
1.20 \mathrm{a} \\
0.50 \mathrm{~b}\end{array}$ & $\begin{array}{l}0.42 \mathrm{f} \\
0.56 \mathrm{e} \\
0.61 \mathrm{~d} \\
0.55 \mathrm{e} \\
0.60 \mathrm{~d} \\
0.70 \mathrm{c} \\
0.76 \mathrm{c} \\
0.78 \mathrm{~b} \\
0.82 \mathrm{~b} \\
1.26 \mathrm{a} \\
0.52 \mathrm{c} \\
\end{array}$ & $\begin{array}{l}0.17 \mathrm{~g} \\
0.21 \mathrm{~cd} \\
0.26 \mathrm{a} \\
0.22 \mathrm{c} \\
0.25 \mathrm{a} \\
0.20 \mathrm{~d} \\
0.24 \mathrm{~b} \\
0.18 \mathrm{f} \\
0.22 \mathrm{c} \\
0.24 \mathrm{~b} \\
0.18 \mathrm{f} \\
\end{array}$ & $\begin{array}{l}0.19 \mathrm{e} \\
0.24 \mathrm{bc} \\
0.28 \mathrm{a} \\
0.24 \mathrm{bc} \\
0.28 \mathrm{a} \\
0.24 \mathrm{bc} \\
0.28 \mathrm{a} \\
0.20 \mathrm{~cd} \\
0.24 \mathrm{bc} \\
0.28 \mathrm{a} \\
0.20 \mathrm{~cd} \\
\end{array}$ & $\begin{array}{l}0.22 \mathrm{f} \\
0.28 \mathrm{c} \\
0.35 \mathrm{~b} \\
0.26 \mathrm{~d} \\
0.30 \mathrm{~b} \\
0.26 \mathrm{~d} \\
0.30 \mathrm{~b} \\
0.23 \mathrm{e} \\
0.28 \mathrm{c} \\
0.30 \mathrm{~b} \\
0.22 \mathrm{ef}\end{array}$ \\
\hline $\begin{array}{l}\text { F test (C) } \\
\text { Bio (B) }\end{array}$ & $\star \star \star ~$ & ** & ** & ** & ** & ** & ** & ** & ** & ** & ** & ${ }_{* *}^{* *}$ \\
\hline$A C \times B$ & ** & ??ח0? & & & & & & $02 * 30$ & 0,39 & & $* *$ & 0033 \\
\hline LSD 5\% $1 \%$ & $\begin{array}{l}0.022 \\
0.030 \\
\end{array}$ & $\begin{array}{l}0.022 \\
0.03\end{array}$ & $\begin{array}{l}0.022 \\
0.03 \\
\end{array}$ & $\begin{array}{l}1.989 \\
2.665\end{array}$ & $\begin{array}{l}1.989 \\
2.665 \\
\end{array}$ & $\begin{array}{r}1.989 \\
2.665 \\
\end{array}$ & $\begin{array}{l}0.039 \\
0.052 \\
\end{array}$ & $\begin{array}{l}0.039 \\
0.052 \\
\end{array}$ & $\begin{array}{l}0.039 \\
0.52\end{array}$ & $\begin{array}{l}0.033 \\
0.046 \\
\end{array}$ & $\begin{array}{l}0.033 \\
0.046 \\
\end{array}$ & $\begin{array}{l}0.033 \\
0.046 \\
\end{array}$ \\
\hline & & Straw- & & & & & & Straw-Ni & & & & \\
\hline $\begin{array}{l}\text { Control } \\
\text { SS3 } \\
\text { SS5 } \\
\text { PM1 } \\
\text { PM2 } \\
\text { PTR1 } \\
\text { PTR2 } \\
\text { (SS+PM)1 } \\
\text { (SS+PM)2 } \\
\text { SSS+PM)3 } \\
\text { SMMF } \\
\text { RMP }\end{array}$ & $\begin{array}{l}2.00 \mathrm{e} \\
4.00 \mathrm{c} \\
5.95 \mathrm{~b} \\
4.00 \mathrm{c} \\
6.00 \mathrm{~b} \\
4.00 \mathrm{c} \\
6.00 \mathrm{~b} \\
4.00 \mathrm{c} \\
6.03 \mathrm{~b} \\
8.00 \mathrm{a} \\
4.00 \mathrm{c} \\
\end{array}$ & $\begin{array}{l}3.95 \mathrm{f} \\
4.90 \mathrm{e} \\
8.95 \mathrm{bc} \\
4.80 \mathrm{e} \\
6.90 \mathrm{~cd} \\
6.00 \mathrm{~d} \\
8.00 \mathrm{c} \\
8.00 \mathrm{c} \\
10.00 \mathrm{~b} \\
12.00 \mathrm{a} \\
7.00 \mathrm{~cd}\end{array}$ & $\begin{array}{r}4.00 \mathrm{~g} \\
8.00 \mathrm{e} \\
12.50 \mathrm{bc} \\
6.00 \mathrm{f} \\
12.00 \mathrm{c} \\
12.00 \mathrm{c} \\
14.00 \mathrm{~b} \\
10.00 \mathrm{~d} \\
12.00 \mathrm{c} \\
16.00 \mathrm{a} \\
8.00 \mathrm{e} \\
\end{array}$ & $\begin{array}{l}14.0 \mathrm{e} \\
18.0 \mathrm{de} \\
22.0 \mathrm{bc} \\
17.0 \mathrm{de} \\
18.0 \mathrm{~cd} \\
22.0 \mathrm{bc} \\
24.0 \mathrm{a} \\
18.0 \mathrm{de} \\
20.0 \mathrm{bc} \\
24.0 \mathrm{a} \\
16.0 \mathrm{e} \\
\end{array}$ & $\begin{array}{l}16.0 \mathrm{de} \\
20.0 \mathrm{bc} \\
22.0 \mathrm{bc} \\
18.0 \mathrm{~cd} \\
20.0 \mathrm{~cd} \\
23.0 \mathrm{ab} \\
25.0 \mathrm{a} \\
20.0 \mathrm{bc} \\
22.0 \mathrm{bc} \\
25.0 \mathrm{a} \\
16.0 \mathrm{e}\end{array}$ & $\begin{array}{l}19.0 \mathrm{bc} \\
22.0 \mathrm{c} \\
24.0 \mathrm{c} \\
20.0 \mathrm{~d} \\
22.0 \mathrm{~d} \\
24.0 \mathrm{~b} \\
26.0 \mathrm{~b} \\
22.0 \mathrm{c} \\
24.0 \mathrm{bc} \\
30.0 \mathrm{a} \\
14.0 \mathrm{~d} \\
\end{array}$ & $\begin{array}{l}5.80 \mathrm{e} \\
6.20 \mathrm{e} \\
6.60 \mathrm{e} \\
7.00 \mathrm{c} \\
7.60 \mathrm{~b} \\
7.00 \mathrm{bc} \\
8.60 \mathrm{a} \\
7.00 \mathrm{~d} \\
7.80 \mathrm{c} \\
8.40 \mathrm{bc} \\
6.60 \mathrm{e}\end{array}$ & $\begin{array}{c}6.00 \mathrm{f} \\
6.60 \mathrm{f} \\
6.00 \mathrm{e} \\
7.40 \mathrm{~d} \\
8.00 \mathrm{c} \\
8.60 \mathrm{~b} \\
9.00 \mathrm{a} \\
7.60 \mathrm{c} \\
8.20 \mathrm{~b} \\
8.800 \mathrm{ab} \\
6.80 \mathrm{c} \\
\end{array}$ & $\begin{array}{l}6.80 \mathrm{e} \\
7.10 \mathrm{~d} \\
7.60 \mathrm{bc} \\
8.20 \mathrm{c} \\
8.60 \mathrm{bc} \\
8.80 \mathrm{~d} \\
10.00 \mathrm{a} \\
8.00 \mathrm{bc} \\
8.80 \mathrm{~b} \\
10.00 \mathrm{a} \\
7.00 \mathrm{c}\end{array}$ & $\begin{array}{l}5.20 \mathrm{~h} \\
6.40 \mathrm{~cd} \\
6.60 \mathrm{c} \\
5.60 \mathrm{~b} \\
7.00 \mathrm{ab} \\
5.80 \mathrm{fg} \\
6.80 \mathrm{~b} \\
6.20 \mathrm{~d} \\
6.80 \mathrm{~b} \\
7.15 \mathrm{a} \\
5.40 \mathrm{~g} \\
\end{array}$ & $\begin{array}{l}6.10 \mathrm{ef} \\
8.20 \mathrm{c} \\
9.20 \mathrm{a} \\
7.20 \mathrm{~d} \\
8.00 \mathrm{c} \\
6.60 \mathrm{ef} \\
7.00 \mathrm{~d} \\
6.80 \mathrm{de} \\
7.80 \mathrm{~cd} \\
9.00 \mathrm{~b} \\
7.00 \mathrm{~d}\end{array}$ & $\begin{array}{l}7.00 \mathrm{~g} \\
10.55 \mathrm{~b} \\
11.60 \mathrm{a} \\
10.60 \mathrm{~b} \\
11.70 \mathrm{a} \\
8.20 \mathrm{e} \\
8.60 \mathrm{~d} \\
8.20 \mathrm{e} \\
9.00 \mathrm{~cd} \\
9.23 \mathrm{c} \\
7.80 \mathrm{f}\end{array}$ \\
\hline $\begin{array}{l}\text { F test (C) } \\
\text { Bio (B) }\end{array}$ & $x^{* *}$ & $x^{* *}$ & $x^{x+}$ & & & & & & & & & \\
\hline ASD $5 \%$ & 1.148 & 1.148 & 1.148 & 2.453 & 2.453 & 2.453 & 0.374 & 0.374 & 0.374 & 0.449 & 0.449 & 0.449 \\
\hline & 1.586 & 1586 & 1586 & 3.255 & 3.255 & 3.255 & 0.504 & 0.504 & 0.504 & 0.604 & 0.604 & 0.604 \\
\hline
\end{tabular}

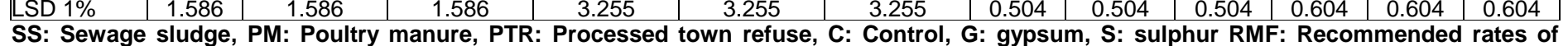
mineral fertilizer. NS not significant ${ }^{\star}$ significant, ${ }^{* \star}$ high significant 
J. Plant Production, Mansoura Univ., Vol. 5(12), December, 2014

Table (12): $\mathrm{Cd}, \mathrm{Cu}, \mathrm{Ni}$ and $\mathrm{Pb}$ contents, ( $\mathrm{mg} \mathrm{kg}^{-1}$ dry weight) of sunflower plants as affected by remedial and amendments.

\begin{tabular}{|c|c|c|c|c|c|c|c|c|c|c|c|c|}
\hline Treatments & \multicolumn{12}{|c|}{ Added agrochemicals } \\
\hline & \multirow{2}{*}{\multicolumn{3}{|c|}{$\frac{G}{\text { Seed-Cd }}$}} & None & G & $\mathbf{S}$ & None & $\mathbf{G}$ & $\mathbf{S}$ & None & $\mathbf{G}$ & $\mathbf{S}$ \\
\hline & & & & \multicolumn{3}{|c|}{ Seed-Cu } & \multicolumn{3}{|c|}{ Seed-Ni } & \multicolumn{3}{|c|}{ Seed-Pb } \\
\hline Control & $0.72 \mathrm{~g}$ & $0.78 \mathrm{~g}$ & $0.75 f$ & $18.0 \mathrm{f}$ & $22.0 \mathrm{f}$ & $20.0 \mathrm{f}$ & $1.83 \mathrm{~h}$ & $2.11 \mathrm{gh}$ & $2.01 \mathrm{~g}$ & $6.92 \mathrm{i}$ & $7.52 \mathrm{f}$ & $7.19 \mathrm{~g}$ \\
\hline SS3 & $0.92 f$ & $1.14 \mathrm{~d}$ & $1.12 \mathrm{c}$ & $24.0 \mathrm{de}$ & $30.0 \mathrm{~d}$ & $28.0 \mathrm{~d}$ & $2.13 \mathrm{f}$ & $2.55 \mathrm{~d}$ & $2.43 \mathrm{c}$ & $8.52 \mathrm{e}$ & $9.55 \mathrm{bc}$ & $8.68 \mathrm{f}$ \\
\hline SS5 & $1.20 \mathrm{~b}$ & $1.30 \mathrm{~b}$ & $1.22 \mathrm{a}$ & $34.0 \mathrm{a}$ & $36.0 \mathrm{~b}$ & $35.0 \mathrm{ab}$ & $2.25 d$ & $2.66 \mathrm{c}$ & $2.54 \mathrm{~b}$ & $9.17 \mathrm{~b}$ & $9.59 \mathrm{bc}$ & $9.15 \mathrm{~d}$ \\
\hline PM1 & $1.04 \mathrm{e}$ & $1.14 \mathrm{~d}$ & $1.08 \mathrm{~cd}$ & $22.0 \mathrm{e}$ & $27.0 \mathrm{e}$ & $26.0 \mathrm{e}$ & $2.51 \mathrm{c}$ & $2.85 \mathrm{ab}$ & $2.66 \mathrm{ab}$ & $7.39 \mathrm{~h}$ & $9.39 \mathrm{c}$ & $8.93 \mathrm{e}$ \\
\hline PM2 & $1.18 \mathrm{~b}$ & $1.24 \mathrm{c}$ & $1.19 b c$ & $30.0 \mathrm{bc}$ & $40.0 \mathrm{a}$ & $34.0 \mathrm{~b}$ & $2.73 \mathrm{a}$ & $2.88 \mathrm{a}$ & $2.74 \mathrm{a}$ & $8.24 f$ & $10.54 \mathrm{a}$ & $9.65 \mathrm{~b}$ \\
\hline PTR1 & $1.14 \mathrm{c}$ & $1.28 \mathrm{~b}$ & $1.02 \mathrm{~d}$ & $24.0 \mathrm{de}$ & $28.0 \mathrm{e}$ & $26.0 \mathrm{e}$ & $2.47 \mathrm{c}$ & $2.62 \mathrm{c}$ & $2.50 \mathrm{bc}$ & $9.14 \mathrm{~b}$ & $9.46 \mathrm{c}$ & $9.40 \mathrm{c}$ \\
\hline PTR2 & $1.24 \mathrm{a}$ & $1.38 \mathrm{a}$ & $1.14 \mathrm{c}$ & $32.0 \mathrm{ab}$ & $38.0 \mathrm{ab}$ & $34.0 \mathrm{~b}$ & $2.61 \mathrm{~b}$ & $2.72 \mathrm{~b}$ & $2.68 \mathrm{ab}$ & $9.67 \mathrm{a}$ & $10.40 \mathrm{a}$ & $9.81 \mathrm{a}$ \\
\hline$(\mathrm{SS}+\mathrm{PM}) 1$ & $0.92 \mathrm{f}$ & $0.96 \mathrm{f}$ & $0.94 \mathrm{c}$ & $26.0 \mathrm{~d}$ & $33.0 \mathrm{~cd}$ & $28.0 \mathrm{~d}$ & $2.05 \mathrm{~g}$ & $2.36 \mathrm{e}$ & $2.08 \mathrm{f}$ & $8.31 \mathrm{f}$ & $9.03 \mathrm{~d}$ & $8.93 \mathrm{e}$ \\
\hline (SS+PM)2 & $1.02 \mathrm{e}$ & $1.20 \mathrm{~d}$ & $1.01 \mathrm{~d}$ & $29.0 \mathrm{c}$ & $34.0 \mathrm{c}$ & $30.0 \mathrm{c}$ & $2.15 \mathrm{e}$ & $2.52 \mathrm{de}$ & $2.19 \mathrm{c}$ & $8.81 \mathrm{~d}$ & $9.28 \mathrm{c}$ & $9.13 \mathrm{~d}$ \\
\hline$(\mathrm{SS}+\mathrm{PM}) 3$ & $1.08 \mathrm{~d}$ & $1.26 \mathrm{c}$ & $1.14 \mathrm{c}$ & $31.0 \mathrm{bc}$ & $40.0 \mathrm{a}$ & $36.0 \mathrm{a}$ & $2.28 \mathrm{~d}$ & $2.59 \mathrm{~cd}$ & $2.37 d$ & $9.03 \mathrm{c}$ & $9.88 \mathrm{~b}$ & $9.55 b c$ \\
\hline RMF & $0.80 \mathrm{e}$ & $0.88 \mathrm{f}$ & $0.92 \mathrm{e}$ & $23.8 \mathrm{e}$ & $29.0 \mathrm{de}$ & $26.0 \mathrm{e}$ & $2.27 \mathrm{~d}$ & 2.33 ef & $2.30 \mathrm{e}$ & $8.04 \mathrm{~g}$ & $8.53 \mathrm{e}$ & $8.45 \mathrm{fg}$ \\
\hline F test (c) & $* *$ & $\star *$ & ${ }^{* *}$ & $* x$ & ** & 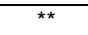 & & $* *$ & $* *$ & & & \\
\hline Bio (B) & $\begin{array}{l}* * \\
* *\end{array}$ & $\begin{array}{l}* * \\
* *\end{array}$ & $\begin{array}{l}* * \\
* *\end{array}$ & $\begin{array}{l}* * \\
* *\end{array}$ & $\begin{array}{l}* * \\
* *\end{array}$ & $\begin{array}{l}* * \\
* *\end{array}$ & ** & ** & $\begin{array}{l}* * \\
* *\end{array}$ & $\begin{array}{l}* * \\
* *\end{array}$ & ** & $\begin{array}{l}* * \\
* *\end{array}$ \\
\hline $\begin{array}{l}A C \times B \\
L S D 5 \%\end{array}$ & 0.032 & 0,032 & 0.032 & 2146 & 2146 & 2146 & 0.052 & 0.052 & 0.052 & 0.145 & 0.145 & 0.145 \\
\hline \multirow[t]{2}{*}{ LSD $1 \%$} & 0.043 & 0.043 & 0.043 & 2.915 & 2.915 & 2.915 & 0.085 & 0.085 & 0.085 & 0.196 & 0.196 & 0.196 \\
\hline & \multicolumn{3}{|c|}{ Leave-Cd } & \multicolumn{3}{|c|}{ Leave-Cu } & \multicolumn{3}{|c|}{ Leave-Ni } & \multicolumn{3}{|c|}{ Leave- $\mathrm{Pb}$} \\
\hline Control & $0.35 f$ & $0.38 \mathrm{~g}$ & $0.36 \mathrm{~g}$ & $32.0 \mathrm{~g}$ & $42.0 \mathrm{~g}$ & $41.5 \mathrm{e}$ & $1.85 \mathrm{~g}$ & $1.92 \mathrm{f}$ & $1.89 \mathrm{~h}$ & $8.26 \mathrm{~g}$ & $9.08 \mathrm{~g}$ & $8.93 \mathrm{f}$ \\
\hline SS3 & $0.51 \mathrm{~b}$ & $0.58 \mathrm{c}$ & $0.52 d$ & $40.0 \mathrm{c}$ & $48.0 \mathrm{e}$ & $440 d$ & $236 \mathrm{~d}$ & $265 \mathrm{c}$ & $2.48 \mathrm{de}$ & $9.14 \mathrm{de}$ & $961 \mathrm{f}$ & $952 \mathrm{e}$ \\
\hline SS5 & $0.53 \mathrm{a}$ & $0.68 \mathrm{a}$ & $055 \mathrm{c}$ & $46.0 \mathrm{~b}$ & $50.0 \mathrm{~d}$ & $50.0 \mathrm{c}$ & $2.53 \mathrm{bc}$ & $2.96 \mathrm{a}$ & $2.84 \mathrm{a}$ & $9.92 \mathrm{a}$ & $11.17 \mathrm{a}$ & $10.86 \mathrm{a}$ \\
\hline PM1 & $0.42 \mathrm{~d}$ & $0.46 \mathrm{f}$ & $0.44 \mathrm{e}$ & $44.0 \mathrm{c}$ & $54.0 \mathrm{c}$ & $51.0 \mathrm{bc}$ & $2.43 \mathrm{~cd}$ & $2.62 \mathrm{c}$ & $2.37 \mathrm{ef}$ & $9.22 \mathrm{~d}$ & $9.70 \mathrm{f}$ & $9.54 \mathrm{e}$ \\
\hline PM2 & $0.48 \mathrm{c}$ & $0.54 \mathrm{~d}$ & $0.49 \mathrm{de}$ & $50.0 \mathrm{a}$ & $58.0 \mathrm{~b}$ & $56.0 \mathrm{a}$ & $2.56 \mathrm{~b}$ & $2.88 \mathrm{a}$ & $2.68 \mathrm{~b}$ & $9.23 d$ & $10.72 \mathrm{~b}$ & $10.63 \mathrm{ab}$ \\
\hline PTR1 & $0.46 \mathrm{~cd}$ & $0.56 \mathrm{~cd}$ & $0.51 \mathrm{~d}$ & $42.0 \mathrm{~d}$ & $51.0 \mathrm{~d}$ & $44.0 \mathrm{~d}$ & $2.47 \mathrm{c}$ & $2.63 \mathrm{c}$ & $2.48 \mathrm{de}$ & $9.06 \mathrm{e}$ & $10.62 \mathrm{~b}$ & $9.59 \mathrm{de}$ \\
\hline $\begin{array}{l}\text { PTR2 } \\
\text { PT }\end{array}$ & $0.48 \mathrm{c}$ & $0.68 \mathrm{a}$ & $0.66 \mathrm{a}$ & $48.0 \mathrm{ab}$ & $56.0 \mathrm{bc}$ & $54.0 \mathrm{ab}$ & $2.60 \mathrm{a}$ & $2.72 \mathrm{~b}$ & $2.63 \mathrm{c}$ & $9.83 \mathrm{ab}$ & $10.91 \mathrm{ab}$ & $10.19 \mathrm{~b}$ \\
\hline$(\mathrm{SS}+\mathrm{PM}) 1$ & $0.40 \mathrm{e}$ & 0.46 ef & $0.42 f$ & 39.0 ef & $48.0 \mathrm{e}$ & $46.0 \mathrm{c}$ & $2.30 \mathrm{e}$ & $2.44 \mathrm{~d}$ & $2.33 \mathrm{f}$ & $9.49 \mathrm{c}$ & $9.90 \mathrm{e}$ & $9.72 \mathrm{~cd}$ \\
\hline (SS+PM)2 & $0.49 \mathrm{bc}$ & $0.48 \mathrm{e}$ & $0.48 \mathrm{de}$ & $42.0 \mathrm{~d}$ & $54.0 \mathrm{c}$ & $49.0 \mathrm{~cd}$ & $2.33 \mathrm{de}$ & $2.50 \mathrm{~d}$ & $2.45 \mathrm{e}$ & $9.59 \mathrm{bc}$ & $10.17 \mathrm{~d}$ & $9.77 \mathrm{~cd}$ \\
\hline (SS+PM) 3 & $0.52 \mathrm{~b}$ & $0.62 \mathrm{~b}$ & $0.55 \mathrm{bc}$ & $48.0 \mathrm{ab}$ & $66.0 \mathrm{a}$ & $50.0 \mathrm{c}$ & $2.41 \mathrm{~cd}$ & $2.67 \mathrm{bc}$ & $2.54 \mathrm{~d}$ & $9.66 \mathrm{~b}$ & $10.30 \mathrm{c}$ & $9.82 \mathrm{c}$ \\
\hline RMF & $0.36 \mathrm{f}$ & $0.47 \mathrm{ef}$ & $0.44 \mathrm{e}$ & $40.0 \mathrm{e}$ & $45.0 \mathrm{f}$ & $42.0 \mathrm{e}$ & $1.98 \mathrm{f}$ & $2.24 \mathrm{e}$ & $2.11 \mathrm{~g}$ & $8.78 \mathrm{f}$ & $9.94 \mathrm{e}$ & $9.71 d$ \\
\hline F test (c & & & & & & 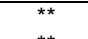 & & 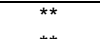 & & & 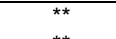 & \\
\hline Bio (B) & ** & ** & ** & $\begin{array}{l}* \star \\
\star \star \\
\star \star\end{array}$ & ** & $\begin{array}{l}* * \\
* *\end{array}$ & ** & ** & ** & $* *$ & ** & ** \\
\hline $\begin{array}{l}\text { AC } \times \text { B } \\
\text { LSD 5\% }\end{array}$ & 0.038 & 0.038 & 0.038 & 2.717 & 2.717 & 2.717 & 0.064 & 0.064 & 0.064 & 0.067 & 0.067 & 0.067 \\
\hline LSD $1 \%$ & 0.051 & 0.051 & 0.051 & 3.626 & 3.626 & 3.626 & 0.086 & 0.086 & 0.086 & 0.090 & 0.090 & 0.090 \\
\hline
\end{tabular}

SS: Sewage sludge, PM: Poultry manure, PTR: Processed town refuse, C: Control, G: gypsum, S: sulphur RMF: Recommended rates of mineral fertilizer. NS not significant ${ }^{*}$ significant, ${ }^{* *}$ high significant 
El-Emary, F. A.A $A^{-1}$ and N.I.A. Talha ${ }^{-2}$

Table (5):Zn, Mn and Fe contents, ( $\mathrm{mg} \mathrm{kg}^{-1}$ dry weight) of pea plants as affected by Remedial and amendments.

\begin{tabular}{|c|c|c|c|c|c|c|c|c|c|}
\hline \multirow[t]{3}{*}{ Treatments } & \multicolumn{9}{|c|}{ Added agrochemicals } \\
\hline & None & $\mathrm{G}$ & $\mathrm{S}$ & None & $G$ & $\mathrm{~S}$ & None & $\mathrm{G}$ & $S$ \\
\hline & \multirow{2}{*}{\multicolumn{3}{|c|}{ Seed-Zn }} & \multicolumn{3}{|c|}{ Seed-Mn } & \multicolumn{3}{|c|}{ Seed-Fe } \\
\hline Control & $20.00 f$ & & $22.00 \mathrm{~g}$ & $9.00 \mathrm{c}$ & $10.60 \mathrm{~b}$ & $10.20 \mathrm{c}$ & $10.90 \mathrm{~d}$ & $16.00 \mathrm{e}$ & $13.00 \mathrm{~d}$ \\
\hline sS3 & $26.00 \mathrm{e}$ & $30.00 \mathrm{~g}$ & 27.00 ef & $10.60 \mathrm{c}$ & $12.00 \mathrm{~cd}$ & $11.40 \mathrm{c}$ & $13.00 \mathrm{c}$ & 15.00 ef & $1425 \mathrm{e}$ \\
\hline SS5 & $34.00 \mathrm{~d}$ & $44.00 \mathrm{~d}$ & $40.00 \mathrm{c}$ & $10.80 \mathrm{c}$ & $13.80 \mathrm{c}$ & $12.40 \mathrm{c}$ & $15.00 \mathrm{c}$ & $17.75 \mathrm{e}$ & $16.00 \mathrm{~d}$ \\
\hline PM1 & $25.00 \mathrm{e}$ & $36.00 \mathrm{f}$ & $30.00 \mathrm{e}$ & $11.00 \mathrm{a}$ & $13.00 \mathrm{~cd}$ & $12.80 \mathrm{~d}$ & $16.60 \mathrm{~cd}$ & $19.00 \mathrm{~cd}$ & $15.50 \mathrm{e}$ \\
\hline PM2 & $40.00 \mathrm{c}$ & $62.00 \mathrm{c}$ & $52.00 \mathrm{c}$ & $11.80 \mathrm{a}$ & $14.40 \mathrm{bc}$ & $13.53 \mathrm{~cd}$ & $16.98 \mathrm{c}$ & $22.00 \mathrm{~b}$ & $17.50 \mathrm{e}$ \\
\hline PTR1 & $42.00 \mathrm{~b}$ & $68.00 \mathrm{~b}$ & $62.00 \mathrm{~d}$ & $13.00 \mathrm{~b}$ & $17.15 \mathrm{c}$ & $15.60 \mathrm{~b}$ & $12.60 \mathrm{~b}$ & 14.20 ef & $13.0 \mathrm{~d}$ \\
\hline PTR2 & $46.00 \mathrm{a}$ & $72.00 \mathrm{~b}$ & $66.00 \mathrm{~d}$ & $16.00 \mathrm{a}$ & $19.60 \mathrm{~b}$ & $17.80 \mathrm{~b}$ & $15.03 \mathrm{a}$ & $22.0 \mathrm{~d}$ & $17.0 \mathrm{a}$ \\
\hline (SS+PM)1 & $22.00 \mathrm{f}$ & $54.00 \mathrm{~g}$ & $30.00 \mathrm{~b}$ & $9.55 \mathrm{c}$ & $10.55 \mathrm{e}$ & $10.20 \mathrm{ab}$ & $12.0 \mathrm{e}$ & $15.25 f$ & $14.20 \mathrm{~b}$ \\
\hline$(\mathrm{SS}+\mathrm{PM}) 2$ & $36.00 \mathrm{~d}$ & $56.00 \mathrm{de}$ & $40.00 \mathrm{ab}$ & $10.65 \mathrm{c}$ & $11.43 \mathrm{e}$ & $10.80 \mathrm{ab}$ & $15.0 \mathrm{e}$ & 18.50 ef & $16.20 \mathrm{~b}$ \\
\hline$(\mathrm{SS}+\mathrm{PM}) 3$ & $52.00 \mathrm{c}$ & $76.50 \mathrm{a}$ & $58.00 \mathrm{a}$ & $11.00 \mathrm{c}$ & $13.00 \mathrm{a}$ & $12.40 \mathrm{a}$ & $18.0 \mathrm{c}$ & $24.0 \mathrm{a}$ & $18.50 \mathrm{~b}$ \\
\hline RMF & $23.00 \mathrm{e}$ & $24.00 \mathrm{i}$ & $25.00 \mathrm{~g}$ & $11.00 \mathrm{~b}$ & $11.55 \mathrm{~b}$ & $11.50 \mathrm{c}$ & $14.0 \mathrm{~d}$ & $18.0 \mathrm{e}$ & $16.00 \mathrm{c}$ \\
\hline F test (c) & $* *$ & $* *$ & ${ }^{* *}$ & $*$ & * & ${ }^{*}$ & ns & $\mathrm{ns}$ & ns \\
\hline Bio (B) & ** & *** & *** & *** & *** & *** & ** & *** & *** \\
\hline$A C \times B$ & ${ }^{\star *}$ & 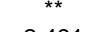 & ** & ** & ** & ** & & ${ }^{* \star}$ & \\
\hline LSD 5\% & 2.491 & 2.491 & 2.491 & 2.352 & 2.352 & 2.352 & 2.723 & 2.723 & 2.723 \\
\hline \multirow[t]{2}{*}{ LSD 1\% } & 3.319 & 3.319 & 3.319 & 3.184 & 3.184 & 3.184 & 3.664 & 3.664 & 3.664 \\
\hline & \multicolumn{3}{|c|}{ Straw-Zn } & \multicolumn{3}{|c|}{ Straw-Mn } & \multicolumn{3}{|c|}{ Straw-Fe } \\
\hline Control & $20.00 \mathrm{de}$ & $21.00 \mathrm{~d}$ & $18.00 \mathrm{f}$ & $30.00 \mathrm{~h}$ & $42.00 \mathrm{i}$ & $33.00 \mathrm{~h}$ & 40.40 ef & 48.80 ef & $44.2 \mathrm{e}$ \\
\hline & $20.00 \mathrm{de}$ & $24.00 \mathrm{~cd}$ & $22.00 \mathrm{~cd}$ & $40.00 \mathrm{~g}$ & $44.00 \mathrm{~g}$ & $42.00 \mathrm{gh}$ & $41.40 \mathrm{f}$ & $43.40 \mathrm{de}$ & $41.65 \mathrm{f}$ \\
\hline SS5 & $28.00 \mathrm{c}$ & $36.00 \mathrm{c}$ & $30.00 \mathrm{bc}$ & $44.00 \mathrm{f}$ & $48.00 \mathrm{~g}$ & $46.00 \mathrm{~g}$ & $48.40 \mathrm{~d}$ & $51.80 \mathrm{c}$ & $50.85 d$ \\
\hline PM1 & $26.00 \mathrm{~d}$ & $30.00 \mathrm{de}$ & $28.25 \mathrm{~cd}$ & $50.00 \mathrm{~b}$ & $60.00 \mathrm{f}$ & $52.00 \mathrm{f}$ & $45.00 \mathrm{~g}$ & $55.20 \mathrm{~h}$ & $49.00 \mathrm{~d}$ \\
\hline PM2 & $28.00 \mathrm{a}$ & $35.00 \mathrm{~d}$ & $29.00 \mathrm{bc}$ & $54.00 \mathrm{e}$ & $75.00 \mathrm{f}$ & $64.00 \mathrm{c}$ & $48.25 \mathrm{~d}$ & $65.60 \mathrm{a}$ & $59.20 \mathrm{c}$ \\
\hline PTR1 & $22.00 \mathrm{~b}$ & $30.50 \mathrm{~b}$ & $25.00 \mathrm{de}$ & $58.00 \mathrm{e}$ & $68.00 \mathrm{e}$ & $60.00 \mathrm{~b}$ & $56.60 \mathrm{c}$ & $74.80 \mathrm{~b}$ & $64.40 \mathrm{~b}$ \\
\hline PTR2 & $30.00 \mathrm{~b}$ & $35.00 \mathrm{a}$ & $32.00 \mathrm{de}$ & $68.00 \mathrm{~b}$ & $80.00 \mathrm{~b}$ & $70.00 \mathrm{a}$ & $63.60 \mathrm{~b}$ & $84.80 \mathrm{~b}$ & $74.00 \mathrm{a}$ \\
\hline$(\mathrm{SS}+\mathrm{PM}) 1$ & $22.00 \mathrm{de}$ & $24.00 \mathrm{~d}$ & $23.00 \mathrm{~cd}$ & $54.00 \mathrm{~d}$ & $64.00 \mathrm{~cd}$ & 60.00 ef & $47.00 \mathrm{de}$ & $53.80 \mathrm{~g}$ & $.50 .80 \mathrm{~g}$ \\
\hline (SS+PM)2 & $26.00 \mathrm{c}$ & $32.00 \mathrm{~b}$ & $28.00 \mathrm{~b}$ & $56.25 \mathrm{c}$ & $70.50 \mathrm{~cd}$ & $66.00 \mathrm{e}$ & $59.45 \mathrm{c}$ & $68.60 \mathrm{f}$ & $61.80 \mathrm{f}$ \\
\hline$(\mathrm{SS}+\mathrm{PM}) 3$ & $28.00 \mathrm{c}$ & $36.00 \mathrm{a}$ & $30.00 \mathrm{a}$ & $65.00 \mathrm{~b}$ & $75.50 \mathrm{bc}$ & $68.00 \mathrm{~b}$ & $63.00 \mathrm{a}$ & $77.80 \mathrm{c}$ & $67.60 \mathrm{e}$ \\
\hline RMF & $22.00 \mathrm{~d}$ & $24.00 \mathrm{c}$ & $23.00 \mathrm{ef}$ & $34.00 \mathrm{~h}$ & $45.00 \mathrm{de}$ & $42.00 \mathrm{~g}$ & $39.40 \mathrm{~g}$ & $45.60 \mathrm{~d}$ & $42.40 \mathrm{f}$ \\
\hline & $* *$ & $* *$ & ** & $* *$ & $* *$ & $* *$ & $* *$ & $* *$ & $* *$ \\
\hline $\mathrm{Bio}(\mathrm{B})$ & ** & ** & *** & *** & ** & ** & *** & *** & ** \\
\hline$A C \times B$ & ${ }^{* *}$ & ** & ** & ** & 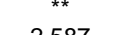 & م507 & ** & 3000 & $\star \star \star ~$ \\
\hline LSD 5\% & 3.038 & 3.038 & 3.038 & 2.587 & 2.587 & 2.587 & 3.099 & 3.099 & 3.099 \\
\hline LSD $1 \%$ & 4.050 & 4.050 & 4.050 & 3.449 & 3.449 & 3.449 & 4.169 & 4.169 & 4.169 \\
\hline
\end{tabular}

$\begin{array}{ll}3.038 & 3.038 \\ 4.050 & 4.050\end{array}$

3.449

2.587

4.169

SS: Sewage sludge, PM: Poultry manure, PTR: Processed town refuse, C: Control, G: gypsum, S: sulphur: RMF: Recommended rates of mineral fertilizer. NS not significant ${ }^{*}$ significant, ${ }^{\star}$ high significant 
Table (13):Yield characteristics of sunflower plants as affected by remedial and amendments

\begin{tabular}{|c|c|c|c|c|c|c|c|c|c|c|c|c|c|c|c|}
\hline \multirow{3}{*}{ Treatments } & \multicolumn{15}{|c|}{ Agrochemicals uses } \\
\hline & None & $\mathbf{G}$ & $\mathbf{S}$ & None & $\mathbf{G}$ & $\mathbf{S}$ & None & $\mathbf{G}$ & $\mathbf{S}$ & \begin{tabular}{l|l} 
None \\
\end{tabular} & G & $\mathbf{S}$ & None & $\mathbf{G}$ & $\mathbf{S}$ \\
\hline & \multicolumn{3}{|c|}{ Seed yield(g)/plant } & \multicolumn{3}{|c|}{ Seed100(g) } & \multicolumn{3}{|c|}{ Seed yield $(\mathrm{mg}) \mathrm{fed}^{-1}$} & \multicolumn{3}{|c|}{ Oil yield $(\mathrm{kg}) \mathrm{fed}^{-1}$} & \multicolumn{3}{|c|}{ Oil \% } \\
\hline Control & $94.27 a$ & 99.99d & $102.56 \mathrm{c}$ & 9.86de & $10.18 \mathrm{bcd}$ & $10.62 d$ & $1.89 f$ & $2.14 f$ & $2.27 \mathrm{e}$ & $556.81 \mathrm{e}$ & $667.02 \mathrm{~cd}$ & $568.39 d$ & & & \\
\hline $\mathrm{SS}_{3}$ & $116.36 \mathrm{bcd}$ & & $127.72 a b c$ & $9.28 f$ & $10.40 \mathrm{bc}$ & & $2.22 d$ & $2.39 \mathrm{cde}$ & $2.40 \mathrm{bc}$ & & & & & & \\
\hline & & & $\frac{a u c c}{a b c}$ & 10 & & 10.9 & $2.42 \mathrm{~b}$ & $2.49 \mathrm{~b}$ & $2.71 \mathrm{~b}$ & & & & & & 27. \\
\hline & & & & & & & $2.41 \mathrm{c}$ & $2.44 b c$ & $2.49 b$ & 656 & & 744. & & $2 g$ & $31.66 c$ \\
\hline & & & & & & & $2.56 a$ & & $2.93 a$ & & & 932. & & & \\
\hline & 99.36de & & & 9.32ef & & $1 \mathrm{~cd}$ & $1.96 \mathrm{ef}$ & $2.13 f$ & $2.39 \mathrm{bcd}$ & 572. & $26 \mathrm{~d}$ & $788.53 a$ & & & $35.03 a$ \\
\hline & $101.76 \mathrm{cde}$ & 114. & $a b c$ & 9.88de & 10.5 & Obcd & $2.03 e$ & $2.29 \mathrm{e}$ & $2.41 \mathrm{bc}$ & 577 & $76 \mathrm{ab}$ & $760.16 a b c$ & & & $31.17 c$ \\
\hline & de & & & $9.74 \mathrm{def}$ & 10 & $4 \mathrm{bcd}$ & $2.15 d$ & $2.24 f$ & 2.29de & & & $723.47 \mathrm{bc}$ & & & $29.44 d$ \\
\hline & & & & $10.52 \mathrm{c}$ & $4 \mathrm{bcd}$ & $83 b c$ & $2.31 \mathrm{c}$ & $2.38 \mathrm{cde}$ & $2.39 \mathrm{cde}$ & 687 & $6 \mathrm{bc}$ & $730.22 a b c$ & & & f $31.31 \mathrm{c}$ \\
\hline & & & & & & & $2.40 \mathrm{c}$ & $2.46 \mathrm{bcd}$ & $2.49 \mathrm{~b}$ & 695 & & $759.42 a$ & & & $28.72 \mathrm{de}$ \\
\hline & $09.12 \mathrm{cde}$ & $9.25 \mathrm{bcd}$ & $9.92 a b c$ & $10.17 \mathrm{~cd}$ & & $10.40 \mathrm{~cd}$ & $2.21 d$ & $2.34 \mathrm{de}$ & $2.39 \mathrm{bcd}$ & $739.09 b c$ & $740.39 b c$ & $764.38 a b$ & $33.81 \mathrm{~b}$ & $33.82 a$ & $33.81 \mathrm{~b}$ \\
\hline $\mathrm{F}$ tes & & & & * & & * & ** & $\star \star$ & ** & Ns & & Ns & $00.01 \mathrm{n}$ & *t. & ** \\
\hline & & & & & & & ** & & ** & & & & & & 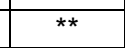 \\
\hline & & & & & & & ** & & & & & & & & \\
\hline & & & & & .520 & .520 & 0.97 & 0.97 & 0.97 & & & 83.884 & 0.992 & & .992 \\
\hline LSD 1\% & 22.303 & 22.303 & 16.64 & 0.696 & 0.696 & 0.696 & 0.131 & 0.131 & 0.131 & 112.972 & 112.972 & 112.972 & 1.343 & 1.343 & 1.343 \\
\hline
\end{tabular}

SS: Sewage sludge, PM: Poultry manure, PTR: Processed town refuse, C: Control, G: gypsum, S: sulphur RMF: Recommended rates of mineral fertilizer-

NS not significant * significant, ${ }^{* \star}$ high significant 
20382039204020412042204320442045204620472048204920502051205220532054205520562057

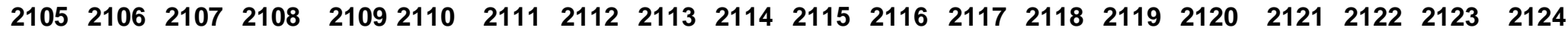
212521262127212821292130

20382039204020412042204320442045204620472048204920502051205220532054205520562057 20382039204020412042204320442045204620472048204920502051205220532054205520562057

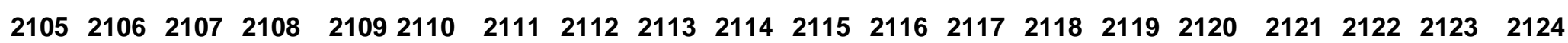
212521262127212821292130

2138 
J. Plant Production, Mansoura Univ., Vol. 5(12), December, 2014 